Submitted to ApJ, 2014 February 10, Accepted 2014 April 28

Preprint typeset using $\mathrm{LATE}_{\mathrm{E}} \mathrm{X}$ style emulateapj v. 04/17/13

\title{
DETECTION OF AN UNIDENTIFIED EMISSION LINE IN THE STACKED X-RAY SPECTRUM OF GALAXY CLUSTERS
}

\author{
Esra Bulbul ${ }^{1,2}$, Maxim Markevitch ${ }^{3}$, Adam Foster ${ }^{1}$, Randall K. Smith $^{1}$ Michael Loewenstein $^{2,4}$, And \\ SCOTT W. RANDALL ${ }^{1}$ \\ ${ }^{1}$ Harvard-Smithsonian Center for Astrophysics, 60 Garden Street, Cambridge, MA, USA \\ ${ }^{2}$ CRESST and X-ray Astrophysics Laboratory, NASA Goddard Space Flight Center, Greenbelt, MD, USA \\ 3 NASA Goddard Space Flight Center, Greenbelt, MD, USA \\ ${ }^{4}$ Department of Astronomy, University of Maryland, College Park, MD, USA
}

Submitted to ApJ, 2014 February 10, Accepted 2014 April 28

\begin{abstract}
We detect a weak unidentified emission line at $E=(3.55-3.57) \pm 0.03 \mathrm{keV}$ in a stacked XMM-Newton spectrum of 73 galaxy clusters spanning a redshift range $0.01-0.35$. MOS and PN observations independently show the presence of the line at consistent energies. When the full sample is divided into three subsamples (Perseus, Centaurus+Ophiuchus+Coma, and all others), the line is seen at $>3 \sigma$ statistical significance in all three independent MOS spectra and the PN "all others" spectrum. The line is also detected at the same energy in the Chandra ACIS-S and ACIS-I spectra of the Perseus cluster, with a flux consistent with XMM-Newton (however, it is not seen in the ACIS-I spectrum of Virgo). The line is present even if we allow maximum freedom for all the known thermal emission lines. However, it is very weak (with an equivalent width in the full sample of only $\sim 1 \mathrm{eV}$ ) and located within $50-110 \mathrm{eV}$ of several known faint lines; the detection is at the limit of the current instrument capabilities and subject to significant modeling uncertainties. On the origin of this line, we argue that there should be no atomic transitions in thermal plasma at this energy. An intriguing possibility is the decay of sterile neutrino, a long-sought dark matter particle candidate. Assuming that all dark matter is in sterile neutrinos with $m_{s}=2 E=7.1 \mathrm{keV}$, our detection in the full sample corresponds to a neutrino decay mixing angle $\sin ^{2}(2 \theta) \approx 7 \times 10^{-11}$, below the previous upper limits. However, based on the cluster masses and distances, the line in Perseus is much brighter than expected in this model, significantly deviating from other subsamples. This appears to be because of an anomalously bright line at $E=3.62 \mathrm{keV}$ in Perseus, which could be an Ar XVII dielectronic recombination line, although its emissivity would have to be 30 times the expected value and physically difficult to understand. In principle, such an anomaly might explain our line detection in other subsamples as well, though it would stretch the line energy uncertainties. Another alternative is the above anomaly in the Ar line combined with the nearby $3.51 \mathrm{keV} \mathrm{K}$ line also exceeding expectation by a factor 10-20. Confirmation with Chandra and Suzaku, and eventually Astro-H, are required to determine the nature of this new line. (APJ HAS THE ABRIDGED ABSTRACT)
\end{abstract}

\section{INTRODUCTION}

Galaxy clusters are the largest aggregations of hot intergalactic gas and dark matter. The gas is enriched with heavy elements (Mitchell et al. (1976); Serlemitsos et al. (1977) and later works) that escape from galaxies and accumulate in the intracluster/intergalactic medium (ICM) over billions of years of galactic and stellar evolution. The presence of various heavy ions is seen from their emission lines in the cluster X-ray spectra. Data from large effective area telescopes with spectroscopic capabilities, such as ASCA, Chandra, XMM-Newton and Suzaku, uncovered the presence of many elements in the $\mathrm{ICM}$, including $\mathrm{O}, \mathrm{Ne}, \mathrm{Mg}, \mathrm{Si}, \mathrm{S}, \mathrm{Ar}, \mathrm{Ca}, \mathrm{Fe}$, and $\mathrm{Ni}$ (for a review see, e.g., Böhringer \& Werner 2010). Recently, weak emission lines of low-abundance Cr and Mn were discovered (Werner et al. 2006; Tamura et al. 2009). Relative abundances of various elements contain valuable information on the rate of supernovae of different types in galaxies (e.g., Loewenstein 2013) and illuminate the enrichment history of the ICM (e.g., Bulbul et al. 2012b).

ebulbul@cfa.harvard.edu
Line ratios of various ions can also provide diagnostics of the physical properties of the ICM and uncover the presence of multi-temperature gas, nonequilibrium ionization states, and nonthermal emission processes such as charge exchange (CX, Paerels \& Kahn 2003).

As for dark matter, 80 yr from its discovery by (Zwicky 1933, 1937), its nature is still unknown (though now we do know for sure it exists, from X-ray and gravitationallensing observations of the Bullet Cluster; Clowe et al. (2006), and we know accurately its cosmological abundance, e.g., Hinshaw et al. (2013)). Among the various plausible dark matter candidates, one that has motivated our present work is the hypothetical sterile neutrino that is included in some extensions to the standard model of particle physics (Dodelson \& Widrow (1994) and later works; for recent reviews see, e.g., Abazajian et al. (2007); Boyarsky et al. (2009)). Sterile neutrinos should decay spontaneously with the rate

$$
\Gamma_{\gamma}\left(m_{s}, \theta\right)=1.38 \times 10^{-29} \mathrm{~s}^{-1}\left(\frac{\sin ^{2} 2 \theta}{10^{-7}}\right)\left(\frac{m_{s}}{1 \mathrm{keV}}\right)^{5},
$$


are unknown but tied to each other in any particular neutrino production model (Pal \& Wolfenstein 1982). The decay of sterile neutrino should produce a photon of $E=m_{s} / 2$ and an active neutrino. The mass of the sterile neutrino may lie in the $\mathrm{keV}$ range, which would place its decay line in the range accessible to X-ray observations of dark matter aggregations, such as clusters of galaxies, nearby galaxies, and the Milky Way (Abazajian et al. 2001a b). So far, searches in various types of massive systems have resulted only in upper limits (e.g., Boyarsky et al. 2012, Abazajian et al. 2012).

Current X-ray archives of XMM-Newton, Chandra and Suzaku contain vast collections of galaxy cluster observations. Mining these databases can result in significant improvement in sensitivity to faint spectral features compared to individual cluster observations (as proposed, e.g., by Abazajian et al. (2001b)), with respect to both the statistical and (in a less obvious way) systematic or instrumental uncertainties. In this paper, we undertake a fishing expedition that combines the spectra of many bright clusters from the XMM-Newton archive in order to search for any kind of faint, unidentified X-ray emission lines - be they thermal lines from previously undetected rare elements in the ICM or the elusive sterile neutrino decay line.

To improve the sensitivity to weak spectral lines and reduce systematic effects, we stack the X-ray spectra from clusters at different redshifts in their rest frame, rescaling the photon energies to $z=0$. After blueshifting each cluster spectrum to $z=0$, any background lines or instrumental response artifacts are smeared out (since they occur in the detector frame), but any weak line intrinsic to clusters would be amplified and may become detectable in the combined spectrum. In this paper, we use this method to detect a previously unknown, extremely faint emission line at $E \approx 3.55-3.57 \mathrm{keV}$. The line was detected in the stacked XMM-Newton X-ray spectra of 73 bright galaxy clusters in the redshift range $0.01<z<0.35$, and independently in several subsamples. The redshift range in our sample corresponds to an energy difference between 0 and $1.2 \mathrm{keV}$ at the energy of the line of $E \approx 3.5 \mathrm{keV}$. In the blueshifted frame, the response features, background, and instrumental lines are smeared by this line shift, while the detected line appears at the same energy, making it possible to detect the 3.57 $\mathrm{keV}$ line with high confidence.

This paper is organized as follows. Sections 2 and 3 describe the XMM-Newton data processing, background modeling, and spectra stacking methods. We also determine the best-fit flux and energy of the detected spectral feature using the XMM-Newton MOS and PN instruments. In Section 4, we confirm the existence of this spectral line in the Chandra ACIS-I and ACIS-S spectra of the Perseus cluster, and obtain an upper limit from the ACIS-I observations of the Virgo Cluster. In Section 5, we discuss the possible interpretations of this new emission line. All errors quoted throughout the paper correspond to $68 \%$ (while in parentheses, 90\%) singleparameter confidence intervals; upper limits are at $90 \%$ confidence, unless otherwise stated.

\section{CLUSTER SAMPLE SELECTION AND DATA ANALYSIS}

\subsection{Sample Selection}

We searched the XMM-Newton archive for galaxy cluster observations that yielded large numbers of X-ray counts. We first selected clusters below a redshift of 0.4 ; higher-redshift clusters are too faint to contribute significantly to the stacked spectrum. We then calculated the total X-ray counts expected from these XMM-Newton observations using the ROSAT count rates reported in eBCS (Ebeling et al. 2000), NORAS (Böhringer et al. 2000), REFLEX (Böhringer et al. 2004), XBACs (Ebeling et al. 1996), and MACS catalogs (Ebeling et al. 2001) and $X M M-N e w t o n$ exposures. To prevent nearby clusters from dominating the stacked spectrum, we used different cluster count limits for different redshift ranges. We chose clusters with a minimum of $10^{5}$ counts per cluster for clusters with $z<0.1$, and $10^{4}$ counts per cluster for clusters with redshifts $0.1<z<0.4$, to have a wide enough range for the redshift-smearing effect. Offset pointings were excluded from the sample. In the end, a sample of 73 clusters was selected. Included in Table 1 are the XMM-Newton observation identification (ObSID) numbers, total MOS and PN clean exposure times, count rates, and our best-fit redshifts (see Section 2.2). The redshift histogram of the sample is given in Figure 1. The count rates reported in Table 1 have been used only for sample selection.

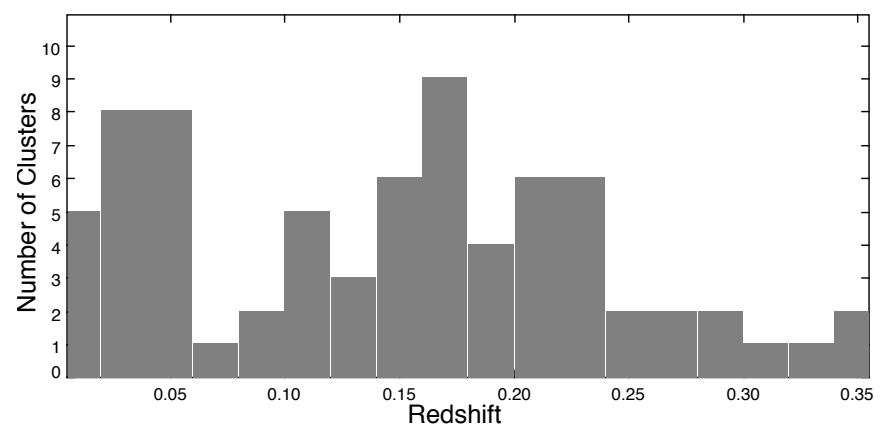

Figure 1. Redshift histogram of the total of 73 galaxy clusters in the sample, selected from the XMM-Newton archive.

\subsection{XMM-Newton Data Analysis}

The EPIC data processing and background modeling were carried out with the XMM-Newton Extended Source Analysis Software (XMM-ESAS; Kuntz \& Snowden 2008; Snowden et al. 2008). We reduced MOS and PN data with the XMM-Newton Science Analysis System $(S A S)$ version 12. Our XMM-Newton analysis is described fully in Bulbul et al. (2012a b); here we give relevant details.

The light-curve filtering was applied to eliminate periods of elevated background. Cleaned events files were created using the good time interval file (GTI) produced by this process. The net exposure time after filtering the event files for good time intervals is given in Table 1 .

Images were created in the $0.4-7.0 \mathrm{keV}$ band for MOS and PN observations and used for detecting point sources with the CIAO tool wavdetect. The images were examined carefully for any missed point sources, as well as for individual MOS CCDs operating in an anomalous state. The CCDs in an anomalous state and all point sources were excluded from further analysis. 
Table 1 XMM-Newton Galaxy Cluster Sample

\begin{tabular}{|c|c|c|c|c|c|c|c|}
\hline Cluster & RA & Decl. & $\overline{\text { ObsID }}$ & $\begin{array}{c}\text { XMM-Newton } \\
\text { MOS Exp } \\
(\mathrm{ks}) \\
\end{array}$ & $\begin{array}{c}\text { XMM-Newton } \\
\text { PN Exp } \\
(\mathbf{k s}) \\
\end{array}$ & $\begin{array}{l}\text { Count Rate } \\
\text { (ROSAT) } \\
\left(\text { cnts s }{ }^{-1}\right) \\
\end{array}$ & $\begin{array}{c}\text { Best-fit } \\
\text { Redshift }\end{array}$ \\
\hline Centaurus & 12: $48: 43.06$ & $-41.0: 18: 42.4$ & $\begin{array}{l}0046340101 \\
0406200101\end{array}$ & 292.0 & 101.9 & 8.8 & $\begin{array}{l}0.009 \\
0.009\end{array}$ \\
\hline A1060 & 10: $36: 46.22$ & $-27.0: 31: 26.9$ & 0206230101 & 67.0 & 24.9 & 3.7 & 0.012 \\
\hline A262 & 1: $52: 45.95$ & 36.0: 9: 14.2 & $\begin{array}{l}0109980101 \\
0504780101 \\
0504780201\end{array}$ & 101.1 & 25.5 & 2.5 & $\begin{array}{l}0.016 \\
0.016 \\
0.016\end{array}$ \\
\hline Perseus & 3: $19: 48.36$ & 41.0: $30: 40.6$ & $\begin{array}{l}0085110101 \\
0305780101\end{array}$ & 317.3 & 38.0 & 35.9 & $\begin{array}{l}0.016 \\
0.016\end{array}$ \\
\hline AWM7 & $2: 54: 32.2$ & $+41: 35: 10$ & $\begin{array}{l}0135950301 \\
0605540101\end{array}$ & 296.2 & 109.7 & 12.7 & $\begin{array}{l}0.016 \\
0.016\end{array}$ \\
\hline Coma & 12: $59: 51.94$ & 27.0: 57: 1.4 & $\begin{array}{l}0124711401 \\
0153750101 \\
0300530101 \\
0300530301\end{array}$ & 171.7 & 66.1 & 25.8 & $\begin{array}{l}0.022 \\
0.022 \\
0.022 \\
0.022\end{array}$ \\
\hline A3581 & 14: $7: 29.68$ & $-27.0: 1: 8.3$ & $\begin{array}{l}0205990101 \\
0504780301 \\
0504780401\end{array}$ & 187.8 & 56.4 & 1.6 & $\begin{array}{l}0.024 \\
0.024 \\
0.023\end{array}$ \\
\hline Ophiuchus & 17: $12: 23.08$ & $-23.0: 22: 44.7$ & 0505150101 & 61.5 & 15.9 & 6.2 & 0.028 \\
\hline A4038 & 23: $47: 41.96$ & $-28.0: 9: 20.1$ & 0204460101 & 56.5 & 23.6 & 3.9 & 0.030 \\
\hline A496 & 4: $33: 39.38$ & $-13.0: 15: 46.5$ & $\begin{array}{l}0135120201 \\
0506260301 \\
0506260401\end{array}$ & 250.2 & 81.1 & 4.1 & $\begin{array}{l}0.033 \\
0.031 \\
0.032\end{array}$ \\
\hline A2063 & 15: 23: 0.96 & 8.0: $36: 25.4$ & $\begin{array}{l}0200120401 \\
0550360101\end{array}$ & 53.8 & 13.6 & 2.3 & $\begin{array}{l}0.034 \\
0.034\end{array}$ \\
\hline A2147 & 16: $2: 21.62$ & 15.0: $58: 35.8$ & $\begin{array}{l}0300350301 \\
0505210601\end{array}$ & 17.6 & 3.3 & 3.2 & $\begin{array}{l}0.035 \\
0.035\end{array}$ \\
\hline A3571 & 13: $47: 22.74$ & $-33.0: 9: 54.3$ & 0086950201 & 48.4 & 14.8 & 5.9 & 0.039 \\
\hline A3558 & 13: $27: 57.2$ & $-31.0: 30: 18.7$ & 0107260101 & 83.0 & 33.1 & 3.5 & 0.048 \\
\hline A 4059 & 23: $57: 2.99$ & $-34.0: 45: 39.6$ & $\begin{array}{l}0109950101 \\
0109950201\end{array}$ & 29.3 & 20.6 & 2.5 & $\begin{array}{l}0.047 \\
0.047\end{array}$ \\
\hline Triangulum Australis & $16: 38: 20.3$ & $-64: 30: 59$ & 0093620101 & 18.4 & & 21.0 & 0.049 \\
\hline Hydra-A & 9: $18: 0.97$ & $-12.0: 5: 11.0$ & $\begin{array}{l}0109980301 \\
0504260101\end{array}$ & 169.4 & 50.9 & 2.6 & $\begin{array}{l}0.052 \\
0.052\end{array}$ \\
\hline A754 & $9: 8: 50.1$ & $-09: 38: 12$ & 0136740101 & 26.8 & 11.0 & 7.5 & 0.054 \\
\hline A2319 & 19: 21: 10.08 & 43.0: 55: 52.6 & $\begin{array}{l}0302150101 \\
0302150201 \\
0600040101\end{array}$ & 159.4 & 50.3 & 4.7 & $\begin{array}{l}0.055 \\
0.054 \\
0.054\end{array}$ \\
\hline Cygnus A & $19: 59: 30.0$ & $+40: 44: 00$ & 0302800201 & 35.5 & 14.1 & 9.3 & 0.056 \\
\hline AS1101 & 23: $13: 59.16$ & $-42.0: 43: 43.8$ & $\begin{array}{l}0123900101 \\
0147800101\end{array}$ & 167.1 & 62.5 & 1.2 & $\begin{array}{l}0.055 \\
0.056\end{array}$ \\
\hline A3112 & $3: 17: 57.44$ & $-44.0: 14: 12.8$ & $\begin{array}{l}0105660101 \\
0603050101 \\
0603050201\end{array}$ & 358.3 & 109.7 & 2.3 & $\begin{array}{l}0.074 \\
0.075 \\
0.075\end{array}$ \\
\hline A 2597 & 23: 25: 17.98 & $-12.0: 6: 23.8$ & $\begin{array}{l}0108460201 \\
0147330101\end{array}$ & 29.9 & 6.3 & 1.6 & $\begin{array}{l}0.081 \\
0.081\end{array}$ \\
\hline A478 & 4: $13: 30.29$ & 10.0: $28: 5.7$ & 0109880101 & 123.0 & 43.1 & 1.6 & 0.083 \\
\hline PKS0745-19 & $7: 47: 31.6$ & $-19.0: 17: 32.7$ & 0105870101 & 315.6 & 5.3 & 0.9 & 0.101 \\
\hline A2811 & $0: 42: 7.06$ & $-28.0: 33: 32.7$ & 0404520101 & 43.8 & 16.8 & 0.8 & 0.108 \\
\hline A2034 & 15: $10: 16.86$ & 33.0: $30: 18.8$ & $\begin{array}{l}0149880101 \\
0303930101 \\
0303930201\end{array}$ & 38.2 & 11.0 & 0.9 & $\begin{array}{l}0.113 \\
0.117 \\
0.117\end{array}$ \\
\hline RXC J0616.8-4748 & 6: $16: 47.14$ & $-47.0: 48: 12.3$ & $\begin{array}{l}0201901101 \\
0302610401\end{array}$ & 43.3 & 16.0 & 0.2 & $\begin{array}{l}0.116 \\
0.113\end{array}$ \\
\hline RXC J0145.0-5300 & 1: $45: 3.38$ & $-53.0: 1: 57.6$ & $\begin{array}{l}0201900501 \\
0404910201 \\
0404911301\end{array}$ & 72.1 & 21.3 & 0.3 & $\begin{array}{l}0.120 \\
0.120 \\
0.120\end{array}$ \\
\hline RXC J1044.5-0704 & 10: $44: 37.64$ & $-7.0: 4: 20.8$ & 0201901501 & 47.9 & 21.3 & 0.5 & 0.130 \\
\hline A1068 & 10: $40: 38.89$ & 39.0: $57: 6.4$ & 0147630101 & 38.3 & 13.2 & 0.7 & 0.138 \\
\hline RXC J2218.6-3853 & $22: 18: 40.22$ & $-38.0: 53: 50.6$ & 0201903001 & 35.5 & 6.8 & 0.4 & 0.140 \\
\hline
\end{tabular}


TABLE 1 - continued from previous page

\begin{tabular}{|c|c|c|c|c|c|c|c|}
\hline Cluster & RA & Decl. & ObsID & $\begin{array}{c}\text { XMM-Newton } \\
\text { MOS Exp } \\
(\mathrm{ks})\end{array}$ & $\begin{array}{c}\text { XMM-Newton } \\
\text { PN Exp } \\
(\mathbf{k s}) \\
\end{array}$ & $\begin{array}{l}\text { Count Rate } \\
\left(\operatorname{cnts~s}^{-1}\right)\end{array}$ & $\begin{array}{r}\text { Best-fit } \\
\text { Redshift }\end{array}$ \\
\hline RXC J0605.8-3518 & $6: 5: 58.31$ & $-35.0: 18: 5.8$ & 0201901001 & 38.3 & 11.6 & 0.4 & 0.132 \\
\hline \multirow[t]{5}{*}{ A1413 } & 11: $55: 24.17$ & 23.0: $24: 17.5$ & 0112230501 & 346.8 & 107.1 & 1.0 & 0.138 \\
\hline & & & 0502690101 & & & & 0.142 \\
\hline & & & 0502690201 & & & & 0.142 \\
\hline & & & 0551280101 & & & & 0.141 \\
\hline & & & 0551280201 & & & & 0.142 \\
\hline \multirow[t]{5}{*}{ A 2204} & 16: $32: 47.11$ & 5.0: $34: 36.1$ & 0112230301 & 124.4 & 35.3 & 0.9 & 0.150 \\
\hline & & & 0306490101 & & & & 0.152 \\
\hline & & & 0306490201 & & & & 0.152 \\
\hline & & & 0306490301 & & & & 0.150 \\
\hline & & & 0306490401 & & & & 0.151 \\
\hline \multirow[t]{2}{*}{ A3888 } & $22: 34: 27.77$ & $-37.0: 44: 59.6$ & 0201903101 & 86.1 & 29.2 & 0.6 & 0.153 \\
\hline & & & 0404910801 & & & & 0.154 \\
\hline \multirow[t]{2}{*}{ RXC J0958.3-1103 } & 9: 58: 17.69 & -11.0: 3: 29.9 & 0201903501 & 17.6 & 2.0 & 0.4 & 0.153 \\
\hline & & & 0404910601 & & & & 0.153 \\
\hline A545 & 5: $32: 20.65$ & -11.0: 33: 8.0 & 0304750101 & 11.3 & 3.7 & 0.4 & 0.154 \\
\hline RXC J2014.8-2430 & 20: $14: 46.14$ & $-24.0: 31: 12.5$ & 0201902201 & 43.1 & 13.5 & 0.6 & 0.155 \\
\hline \multirow[t]{3}{*}{ RX J1720.1+2638 } & 17: $20: 4.81$ & 26.0: $37: 30.3$ & 0500670201 & 108.8 & 29.9 & 0.8 & 0.160 \\
\hline & & & 0500670301 & & & & 0.158 \\
\hline & & & 0500670401 & & & & 0.159 \\
\hline \multirow[t]{3}{*}{ RXC J0645.4-5413 } & $6: 45: 25.63$ & $-54.0: 12: 11.2$ & 0201901201 & 35.5 & 6.7 & 0.5 & 0.167 \\
\hline & & & 0201903401 & & & & 0.167 \\
\hline & & & 0404910401 & & & & 0.167 \\
\hline A1201 & 11: $12: 58.9$ & 13.0: $26: 19.5$ & 0500760101 & 51.5 & 41.8 & 0.4 & 0.169 \\
\hline A1914 & $14: 26: 7.37$ & 37.0: 49: 29.9 & 0112230201 & 25.5 & 9.2 & 1.2 & 0.166 \\
\hline A 2345 & $21: 27: 11.16$ & $-12.0: 9: 21.5$ & 0604740101 & 93.0 & 24.6 & 0.4 & 0.180 \\
\hline \multirow[t]{3}{*}{ A 2218} & $16: 35: 41.82$ & $66.0: 11: 37.6$ & 0112980101 & 98.7 & 24.9 & 0.4 & 0.176 \\
\hline & & & 0112980401 & & & & 0.176 \\
\hline & & & 0112980501 & & & & 0.176 \\
\hline $\mathrm{A} 2254$ & $17: 17: 41.32$ & 19.0: 40: 8.0 & 0601080101 & 111.7 & 39.2 & 0.3 & 0.178 \\
\hline \multirow[t]{2}{*}{ A665 } & $8: 30: 58.0$ & $65.0: 50: 20.0$ & 0109890401 & 43.3 & & 0.6 & 0.182 \\
\hline & & & 0109890501 & & & & 0.180 \\
\hline A1689 & 13: 11: 33.68 & $-1.0: 20: 29.7$ & 0093030101 & 69.3 & 26.5 & 1.0 & 0.183 \\
\hline A383 & $2: 48: 6.7$ & $-3.0: 30: 57.9$ & 0084230501 & 52.9 & 16.1 & 0.3 & 0.186 \\
\hline A520 & 4: $54: 13.14$ & 2.0: $55: 55.0$ & 0201510101 & 59.1 & 12.1 & 0.4 & 0.199 \\
\hline \multirow[t]{2}{*}{ A2163 } & $16: 15: 41.47$ & $-6.0: 9: 16.7$ & 0112230601 & 20.1 & 6.3 & 0.9 & 0.203 \\
\hline & & & 0112231501 & & & & 0.203 \\
\hline A209 & 1: $31: 50.59$ & $-13.0: 37: 36.4$ & 0084230301 & 33.9 & 11.3 & 0.5 & 0.210 \\
\hline A963 & 10: $17: 9.38$ & 39.0: 2: 49.9 & 0084230701 & 44.0 & 13.1 & 0.4 & 0.203 \\
\hline RXC J1504.1-0248 & $15: 4: 7.79$ & $-2.0: 48: 11.8$ & 0401040101 & 72.1 & 27.1 & 1.0 & 0.215 \\
\hline MS $0735.7+7421$ & 7: $41: 28.18$ & 74.0: $14: 25.1$ & 0303950101 & 92.7 & 37.2 & 0.2 & 0.2165 \\
\hline A773 & $9: 17: 45.67$ & 51.0: $43: 33.2$ & 0084230601 & 26.7 & 14.2 & 0.5 & 0.219 \\
\hline AS0592 & 6: $38: 39.55$ & $-53.0: 57: 59.1$ & 0650860101 & 56.2 & 7.5 & 0.3 & 0.220 \\
\hline A2390 & 21: 53: 39.66 & 17.0: $42: 45.5$ & 0111270101 & 19.9 & 8.2 & 0.5 & 0.227 \\
\hline A 2667 & $23: 51: 42.3$ & $-26.0: 4: 13.6$ & 0148990101 & 38.6 & 14.0 & 0.5 & 0.230 \\
\hline $\mathrm{A} 267$ & 1: $52: 38.71$ & $0.0: 59: 49.4$ & 0084230401 & 11.7 & & 0.4 & 0.230 \\
\hline RX J2129.6+0005 & 21: $29: 34.73$ & $0.0: 4: 53.7$ & 0093030201 & 63.0 & 18.1 & 0.3 & 0.235 \\
\hline \multirow[t]{2}{*}{ RXC J1314.4-2515 } & 13: $14: 35.3$ & $-25.0: 15: 38.7$ & 0501730101 & 64.7 & 19.8 & 0.3 & 0.247 \\
\hline & & & 0551040101 & & & & 0.247 \\
\hline \multirow[t]{3}{*}{ A1835 } & 14: $1: 1.88$ & 2.0: $52: 40.4$ & 0147330201 & 241.8 & 62.4 & 1.0 & 0.253 \\
\hline & & & 0551830101 & & & & 0.250 \\
\hline & & & 0551830201 & & & & 0.252 \\
\hline A1758 & 13: $32: 48.01$ & 50.0: $32: 0.9$ & 0142860201 & 58.9 & 17.7 & 0.3 & 0.279 \\
\hline A 1763 & 13: $35: 24.76$ & 40.0: 59: 41.2 & 0084230901 & 24.1 & 8.2 & 0.6 & 0.222 \\
\hline A689 & $8: 37: 20.93$ & 14.0: $58: 6.8$ & 0501010101 & 69.3 & 26.5 & 0.5 & 0.279 \\
\hline \multirow[t]{3}{*}{ ZW 3146} & 10: $23: 39.84$ & 4.0: $11: 5.6$ & 0108670101 & 387.6 & 146.3 & 0.5 & 0.290 \\
\hline & & & 0605540201 & & & & 0.291 \\
\hline & & & 0605540301 & & & & 0.289 \\
\hline A781 & 9: $20: 9.6$ & 30.0: $29: 47.3$ & 0150620201 & 118.9 & 47.5 & 0.3 & 0.290 \\
\hline
\end{tabular}


TABLE 1 - continued from previous page

\begin{tabular}{|c|c|c|c|c|c|c|c|}
\hline Cluster & RA & Decl. & ObsID & $\begin{array}{c}\text { XMM-Newton } \\
\text { MOS Exp } \\
(\mathbf{k s}) \\
\end{array}$ & $\begin{array}{c}\text { XMM-Newton } \\
\text { PN Exp } \\
(\mathbf{k s}) \\
\end{array}$ & 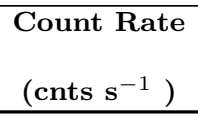 & $\begin{array}{r}\text { Best-fit } \\
\text { Redshift }\end{array}$ \\
\hline & & & 0401170101 & & & & 0.295 \\
\hline 1E $0657-558$ & 06: 58: 31.1 & $-55: 56: 49$ & 0112980201 & 43.7 & 17.4 & 4.1 & 0.296 \\
\hline \multirow[t]{2}{*}{ MS 2137.3-2353 } & 21: $40: 15.28$ & $-23.0: 39: 43.5$ & 0008830101 & 21.4 & 6.3 & 0.2 & 0.313 \\
\hline & & & 0673830201 & & & & 0.313 \\
\hline MACS J2229.7-2755 & 22: $29: 45.13$ & $-27.0: 55: 33.7$ & 0651240201 & 58.3 & 14.0 & 0.2 & 0.320 \\
\hline \multirow[t]{2}{*}{ MACS J1532.8+3021 } & 15: $32: 48.98$ & 30.0: 21: 14.8 & 0039340101 & 21.5 & 8.0 & 0.2 & 0.350 \\
\hline & & & 0651240101 & & & & 0.345 \\
\hline AS1063 & 22: $48: 46.69$ & $-44.0: 30: 48.9$ & 0504630101 & 21.6 & 18.0 & 0.4 & 0.354 \\
\hline
\end{tabular}

Subtle errors in the detector energy gain may cause a fraction of a percent shifts of the location of the emission lines in different X-ray observations of the same cluster. In addition, a redshift measured from the optical observations of a cluster may differ from an X-ray redshift of the gas. To be able to stack spectra in the same frame, we determined the best-fit X-ray redshift for each XMM-Newton observation using the bright Fe lines. These redshifts (Table 1), which correct for both of the above-mentioned effects, were then used to scale the individual spectra in the source frame of each observation, as will be described in Section 2.3. Our selected observations provide adequate statistics to fit an X-ray redshift for each spectrum.

For most clusters, the spectra were extracted within the overdensity radius $R_{500}$. The overdensity radii were calculated using the Vikhlinin et al. (2009) masstemperature scaling relation for each cluster. Due to the large solid angle of nearby clusters, e.g., Coma, Perseus and Centaurus, their spectra were extracted within the full field of view (FOV). Redistribution matrix files (RMFs) and ancillary response files (ARFs) were created with the $S A S$ tools rmfgen and arfgen, respectively.

Although we stack the cluster spectra in this work (and end up using only the $2-10 \mathrm{keV}$ band for the line search), it is still important to accurately subtract the background from each individual observation. For each extracted spectrum, we model a superposition of four main background components: quiescent particle background (QPB), cosmic X-ray background emission (including Galactic halo, local hot bubble (LHB), and unresolved extragalactic sources), solar wind CX as well as residual contamination from soft protons. We use the ROSAT All-Sky Survey (RASS) background spectrum to model the soft X-ray background using the background tool at the High Energy Astrophysics Science Archive Research Center Web site. The RASS spectrum was extracted from an annulus from $1^{\circ}$ to $2^{\circ}$ surrounding the cluster center, with the assumption that this spectrum reasonably represents the soft X-ray background in the direction of the cluster.

We simultaneously modeled the soft X-ray emission from theLHB or heliosphere with a cool unabsorbed single-temperature thermal component $(E \sim 0.1 \mathrm{keV})$, while the Galactic hotter halo and intergalactic medium were modeled with an absorbed thermal component ( $E \sim 0.2 \mathrm{keV})$. The energies of the apec model were restricted but allowed to vary with free normalizations. The abundances were set to $1 A_{\odot}$. We model the contamination due to unresolved point sources using an absorbed power-law component with a spectral index of $\alpha \simeq 1.46$ and normalization of $8.88 \times 10^{-7}$ photons $\mathrm{keV}^{-1} \mathrm{~cm}^{-2}$ $\mathrm{s}^{-1}$ at $\sim 1 \mathrm{keV}$ (Kuntz \& Snowden 2008). Soft-proton flares are largely removed by the light-curve filtering. However, after the filtering some soft-proton residuals may remain in the data and were modeled by including an extra power-law model component and diagonal response matrices provided in the $S A S$ distribution in the final spectral analysis (Snowden et al. 2008).

The EPIC-MOS QPB spectra have two bright instrumental fluorescent lines: the Al-K (1.49 keV) and the Si-K (1.74 keV) lines. The PN QPB spectra have fluorescent lines of Al-K (1.49 keV), Ni-K (7.48 keV), Cu-K $(8.05,8.91 \mathrm{keV})$, and Zn-K $(8.64,9.57 \mathrm{keV})$. Since small variations in the gain and the line strengths between the source and background spectra can lead to residuals in the spectral fitting (Kuntz \& Snowden 2008) and XMMESAS software does not include these instrumental lines in the QPB spectra, we modeled these instrumental lines spectrally by adding Gaussian models to our spectral fits to determine the best-fit energies, widths, and normalizations. The total background was constructed by adding the models for the Al-K, Si-K, Ni-K, Cu-K, and Zn-K lines with the best-fit energies, widths, and normalizations to the QPB produced in the XMM-ESAS analysis for all pointings. These total QBP spectra were directly subtracted from the summed observation to obtain source spectra.

The fitting of the source spectra was done with the spectral fitting package XSPEC 12.8.0 (Arnaud 1996). The $0.3-10 \mathrm{keV}$ energy interval was used for MOS spectra, whereas the $0.4-10.0 \mathrm{keV}$ band was used for the PN fits. To determine the best-fit cluster redshifts for each observation (given in Table 1), the cluster spectra were fit with a standard absorbed multi-temperature collisional equilibrium plasma model (apec; Smith et al. 2001) and AtomDB v2.0.2 (Foster et al. 2012). We did not observe any differences beyond a fraction of a percent in terms of the detector gain variations.

\subsection{Spectra Stacking Methods}

The best way of distinguishing a real spectral feature in a class of distant objects from instrumental artifacts and the X-ray background features is to detect that feature in multiple objects at different redshifts in their rest frame, 
in which case the line coming from an object will stay at the same energy, unlike the detector artifacts. To accomplish this, we stacked the spectra of our selected 73 clusters, blueshifting them to the source frame using the best-fit X-ray redshift of each observation determined above.

Technically, the energies of the source and background $\mathrm{X}$-ray events were rescaled to the source frame using the best-fit redshifts. The scaled event files were then used to extract the source within $r=R_{500}$ or the full FOV of MOS, and the same extraction region was used for PN observations for nearby clusters that fill the FOV. The particle background spectra were extracted using the scaled filter wheel closed data. Counts from each individual spectrum were co-added into a single stacked spectrum using the FTOOL mathpha to produce the stacked source and the particle background spectra. At the end of the stacking process, we obtained spectra with $\sim 6$ Ms of good cluster exposure with MOS 1 and MOS 2 (that were co-added) and $\sim 2 \mathrm{Ms}$ with $\mathrm{PN}$ for the full XMM-Newton sample.

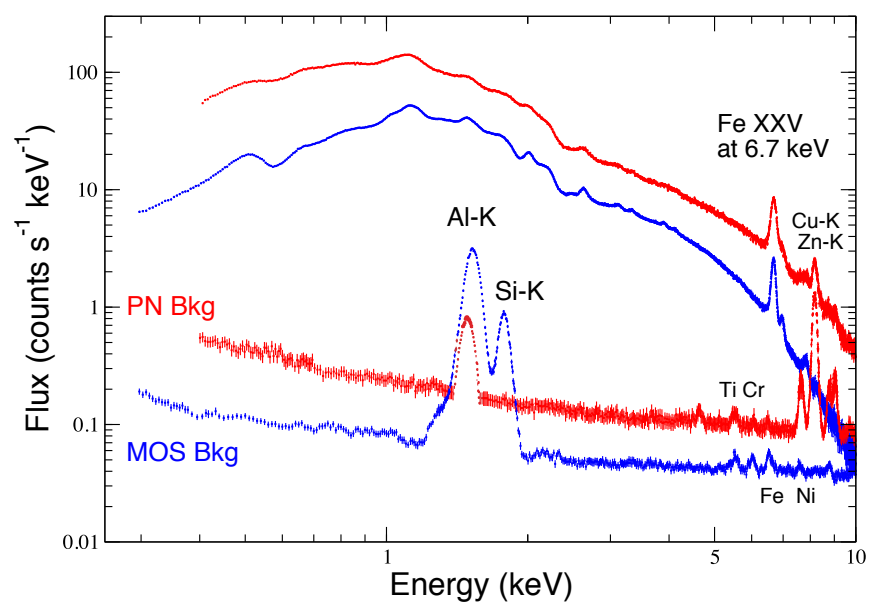

Figure 2. XMM-Newton MOS and PN background-subtracted source spectra and particle background spectra for the Perseus Cluster. The spectra were obtained by co-adding the observations of the cluster in the cluster's rest frame. In the co-added scaled spectra, the Fe Xxv line is located at its rest energy, $\sim 6.7 \mathrm{keV}$. Energy of background and instrumental lines is blue-shifted according to the cluster's redshift.

The RMF and ARF to be used with the stacked spectrum were constructed by averaging the responses for individual observations with proper weighting. The individual RMFs and ARFs were first remapped to the source frame using the best-fit redshifts. The weighing factors for stacking RMFs and ARFs were calculated using the total counts in the energy band we will use for our line search $(2-10 \mathrm{keV})$. These factors $\left(\omega_{\mathrm{cnt}}\right)$ are given in Table 4 . The weighted and normalized ARFs and RMFs were stacked using the FTOOLS addarf and addrmf. These X-ray count-weighted response files were used to model the continuum and the known plasma emission lines; we will also try a different weighting of responses for the possibly nonthermal new line, as will be described below.

For a check, each background-subtracted, blue-shifted, single-cluster spectrum was fit with an apec model using the corresponding scaled ARF and RMF to verify that the best-fit redshifts were consistent with zero. For illustration, the co-added MOS and PN source and background spectra of the Perseus Cluster in its source frame are shown in Figure 2. We note that the Fe Xxv line is located at its rest energy $\sim 6.7 \mathrm{keV}$, while the background and instrumental lines are blue-shifted.

The stacked MOS and PN source and background spectra of the clusters in the sample are shown in Figure 3 The background spectra show the smearing effect on the background lines, e.g., Al-K (1.48 keV), Si-K (1.75 keV), Cr $(5.4 \mathrm{keV}), \mathrm{Mn}(5.8 \mathrm{keV}), \mathrm{Fe}-\mathrm{K}(6.4 \mathrm{keV}), \mathrm{Cu}-\mathrm{K}(8.05$ $\mathrm{keV}, 8.91 \mathrm{keV}), \mathrm{Zn}-\mathrm{K}(8.64 \mathrm{keV}, 9.61 \mathrm{keV})$ and $\mathrm{Au}(9.1$ $\mathrm{keV})$. They are much less prominent in the stacked spectrum compared with the single-source spectrum shown in Figure 2. Similarly, any residuals from inaccurate background subtraction are smeared. We will see other advantages of this smearing below.

\section{ANALYZING THE STACKED XMM-NEWTON SPECTRA}

We will limit our line search to the $2-10 \mathrm{keV}$ energy band. After looking at the stacked spectra, we concluded that the band below $2 \mathrm{keV}$ is hopelessly crowded with lines, such as the strong Ne $\mathrm{x}(1.21 \mathrm{keV})$, Fe XxIV $(1.55 \mathrm{keV}), \mathrm{Mg}$ XII $(1.74 \mathrm{keV})$, and Si XII $(1.86 \mathrm{keV})$ features, making the detection of any weak emission features between them difficult, given the $\sim 100 \mathrm{eV}$ energy resolution of XMM-Newton and other CCD detectors.

To search for any unidentified spectral lines in the stacked spectra, we need to model the known lines and the continuum emission to a very good precision. We do not necessarily need to obtain a physically meaningful model (which would be a mixture of all the thermal components in all the clusters), but one that allows enough freedom to account for all known lines and the possible errors in their theoretical emissivities. To this end, we fit the background-subtracted stacked source spectra with a line-free multi-temperature apec model to represent the continuum emission with high accuracy, and then we add individual lines. We start with four continuum components to represent the multi-temperature nature of the stacked spectra. The line-free apec model accounts for the continuum due to thermal bremsstrahlung, radiative recombination, and two-photon emissions. The best-fit temperature and normalization parameters of line-free apec models are shown in Table 2 . The best-fit temperatures in the table do not have physical meaning, since they are obtained by fitting the stacked blue-shifted spectra. (We note that the continuum of a redshifted thermal model can be well represented by a continuum with a different redshift and a different temperature.) The abundance was set to 0.3 in order to include the recombination edges in the fitting process. The abundance parameter does not affect the line modeling, since the line-free apec model does not include lines.

In order to account for the known plasma emission lines in a model-independent way, for each known line in the $2.0-10.0 \mathrm{keV}$ band, we added a Gaussian line to the model. Initially we have added Gaussian models for the known strong emission lines from the AtomDB databas ${ }^{1}$ with emissivities $>5 \times 10^{-19}$ photons $\mathrm{cm}^{3} \mathrm{~s}^{-1}$ for the lowest temperature given in Table 2. The strong emission lines (which can be resolved with a CCD detector)

\footnotetext{
${ }^{1}$ http://www.atomdb.org/Webguide/webguide.php
} 

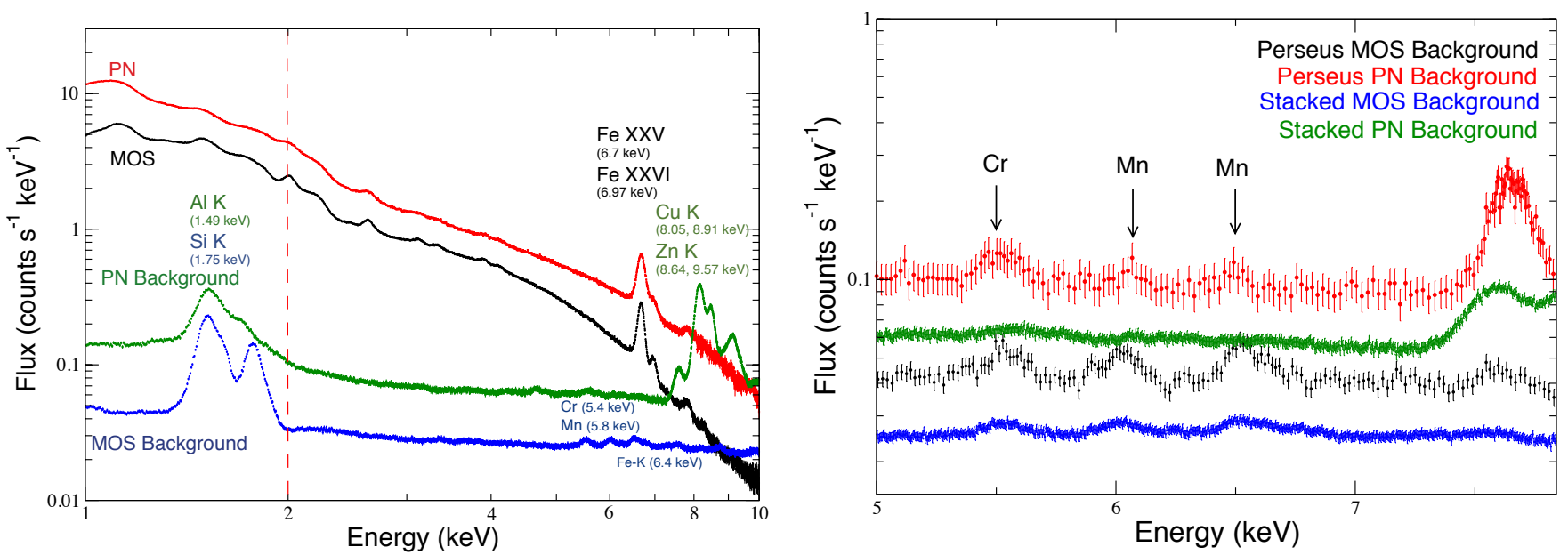

Figure 3. Left Panel: stacked XMM-Newton MOS and PN background-subtracted source spectra and particle background spectra of the full sample. The spectrum of each observation was scaled to the rest frame prior to stacking. The total filtered exposure time was 6 Ms for MOS and $2 \mathrm{Ms}$ for PN. The background MOS (in blue) and PN (in green) spectra show the effect of smearing of instrumental lines, such as $\mathrm{Cr}, \mathrm{Mn}, \mathrm{Fe}$, and $\mathrm{Ni}$, as well as Al-K and Si-K fluorescent lines. The effect is due to the stacking of background spectra that are scaled by different cluster redshifts. Right Panel: close-up view of $5.0-8.0 \mathrm{keV}$ band of the background XMM-Newton MOS and PN spectra of the Perseus Cluster compared to the stacked XMM-Newton MOS and PN background spectra. The background lines are less prominent in the stacked background spectra than in the single-source background spectra.

included in our model at their rest energies are $\mathrm{Al}$ XIII (2.05 keV), Si XIV (2.01 keV and $2.51 \mathrm{keV})$, Si XII (2.18 $\mathrm{keV}, 2.29 \mathrm{keV}$, and $2.34 \mathrm{keV}), \mathrm{S}$ XIV $(2.62 \mathrm{keV}), \mathrm{S}$ XV (complex at $2.45 \mathrm{keV}, 2.88 \mathrm{keV}$ ), Ar XVII (triplet at 3.12 $\mathrm{keV}, 3.62 \mathrm{keV}, 3.68 \mathrm{keV}), \mathrm{K}$ XVIII $(3.47 \mathrm{keV}$ and 3.51 $\mathrm{keV}), \mathrm{K}$ XIX (3.71 keV), Ca XIX (complex at $3.86 \mathrm{keV}$, $3.90 \mathrm{keV}, 4.58 \mathrm{keV})$, Ar XVIII (3.31 keV, $3.93 \mathrm{keV})$, Ca Xx $(4.10 \mathrm{keV})$, Cr XXIII $(5.69 \mathrm{keV})$, Fe XXIV (complex at 6.62 $\mathrm{keV}$ ), Fe XxV (complex at $6.70 \mathrm{keV}, 8.29 \mathrm{keV}, 7.81 \mathrm{keV}$, $7.88 \mathrm{keV})$, Fe XXVI (6.95 keV, $8.3 \mathrm{keV}$, and $8.70 \mathrm{keV})$, and Ni XXVII $(7.79 \mathrm{keV})$. Initially, a total of 28 Gaussian model components were included in the $2-10 \mathrm{keV}$ energy band. Individual Gaussian components were then removed if they were not required to accurately model the spectra (to improve convergence of the fit). The widths of Gaussians were left free, but restricted to the range 0 $<\Delta E / E<10^{-2}$. The energies of the Gaussian components were allowed to vary by up to $5 \mathrm{eV}$ to account for residual uncertainties in the gain and in the energies in the atomic database. This way, we were able to model the continuum emission and strong known emission lines accurately, leaving a clean residual spectrum to search for any unidentified lines.

We also fit a power-law model in the full band to represent the residual soft-proton background contamination (see Section 2.2), and we used these power law indices and normalizations for further narrower-band fits (see Section 3.1). The spectral counts in each energy bin were sufficiently high to allow the use of the Gaussian statistics in this analysis (Protassov et al. 2002).

\subsection{Stacked Spectra of the Full Cluster Sample}

After the stacking process, we obtained a total of $8.5 \times 10^{6}$ source counts in the 6 Ms MOS spectra, while the $2 \mathrm{Ms}$ PN stacked spectra have a total of $5.1 \times 10^{6}$ source counts. The line-free apec model with Gaussian lines produces an acceptable fit to the stacked MOS and PN spectra with $\chi^{2}$ values of 564.8 for 566 dof (MOS) and 510.5 for 564 degrees of freedom (dof) (PN). After modeling all the known thermal plasma lines in the stacked spectrum, we examined the residuals in each 1 $\mathrm{keV}$ band carefully. We found one significant unidentified residual emission feature at $E \approx 3.55-3.57 \mathrm{keV}$, which is not associated with any plasma emission lines in the band. Near this line, there are four tabulated weak thermal emission lines of $\mathrm{K}$ XVIII $\left(1 \mathrm{~s}^{1} 2 \mathrm{~s}^{1} \rightarrow 1 \mathrm{~s}^{2}\right)$ at a rest energy of $3.47 \mathrm{keV}, \mathrm{K}$ XVIII $\left(1 \mathrm{~s}^{1} 2 \mathrm{p}^{1} \rightarrow 1 \mathrm{~s}^{2}\right)$ at 3.51 $\mathrm{keV}$, a dielectronic recombination (DR) line of Ar XVII at $3.62 \mathrm{keV}, \mathrm{Ar}$ XVII $\left(1 \mathrm{~s}^{1} 3 \mathrm{p}^{1} \rightarrow 1 \mathrm{~s}^{2}\right)$ at $3.68 \mathrm{keV}$, and $\mathrm{K}$ XIX $\left(2 \mathrm{p}^{1} \rightarrow 1 \mathrm{~s}^{1}\right)$ at $3.72 \mathrm{keV}$.

In order to separate the excess emission feature from these weak contaminating $\mathrm{K}$ and Ar lines, we make conservative estimates of their flux using AtomDB. Ideally, line flux measurements would be based on other lines of the same ions; however, there are no other strong K XVIII and K XIX lines in the spectrum. Therefore, we use the lines from the relatively clean part of the band, namely, the S XVI $\left(2 \mathrm{p}^{1} \rightarrow 1 \mathrm{~s}^{1}\right)$, Ca XIX $\left(1 \mathrm{~s}^{1} 2 \mathrm{p}^{1} \rightarrow 1 \mathrm{~s}^{2}\right)$, and Ca $\mathrm{xx}\left(2 \mathrm{p}^{1} \rightarrow 1 \mathrm{~s}^{1}\right)$ lines at $2.63 \mathrm{keV}, 3.90 \mathrm{keV}$, and 4.11 $\mathrm{keV}$, respectively, to estimate the flux of the $3.47 \mathrm{keV}$, $3.51 \mathrm{keV}, 3.68 \mathrm{keV}$ and $3.72 \mathrm{keV}$ lines. The best-fit flux measurements of these S XVI, Ca XIX, and Ca Xx lines are given in Table 2 .

We assume that the relative abundances of $\mathrm{S}, \mathrm{Ca}, \mathrm{Ar}$, and $\mathrm{K}$ are proportional to their abundances in the solar photosphere (Anders \& Grevesse 1989). While this may not be exactly true, it gives a reasonable starting point (we will relax this assumption below). Then, using AtomDB, we calculated the relative emissivity of the $\mathrm{K}$ XVIII, K XIX, and Ar XVII lines compared to the S XVI, Ca XIX, and Ca XX lines based on the equilibrium collisional plasma conditions at the various temperatures of our line-free apec components. In practice, the emissivities of K XVIII, K XIX, and Ar XVII lines are stronger at the lowest temperatures of each model, so the other components can be ignored. The curves in Figure 4 represent the emissivities of $\mathrm{K}$ and Ar lines as a function of plasma temperature for the normalizations of the lowest temperature components measured in our spectra.

Having obtained the relative theoretical emissivity of 
Table 2

Best-fit Temperature and Normalizations of line-free apec Model in $2-10 \mathrm{keV}$ Fit to the Stacked MOS and PN Spectra for Various Samples. The temperature $\left(k T_{i}\right)$ normalization $\left(N_{i}\right)$ are in the units of $\mathrm{keV}$ and $\left(10^{-2} \mathrm{~cm}^{-5}\right)$, respectively and the line fluxes of $\mathrm{S}$ XVI, $\mathrm{Ca} \mathrm{XIX}$, and Ca Xx are in the units of $10^{-5}$ photons $\mathrm{cm}^{-2} \mathrm{~s}^{-1}$ at rest energies $2.63 \mathrm{keV}, 3.90 \mathrm{keV}$, and $4.11 \mathrm{keV}$, respectively.

\begin{tabular}{|c|c|c|c|c|c|c|c|c|}
\hline Parameters & $\begin{array}{c}\text { Full } \\
\text { Sample }\end{array}$ & $\mathrm{PN}$ & $\begin{array}{c}\text { Coma } \\
+ \text { Centaurus } \\
+ \text { Ophiuchus } \\
\text { MOS }\end{array}$ & $\mathrm{PN}$ & $\begin{array}{l}\text { Excluding } \\
\text { Nearby } \\
\text { Clusters } \\
\text { MOS }\end{array}$ & $\mathrm{PN}$ & Perseus & $\mathrm{PN}$ \\
\hline $\mathrm{kT}_{1}$ & $5.9 \pm 0.1$ & $7.3 \pm 0.2$ & $3.9 \pm 0.1$ & $2.5 \pm 0.2$ & $3.5 \pm 0.2$ & $2.0 \pm 0.3$ & $3.6 \pm 0.6$ & $2.17 \pm 0.9$ \\
\hline$N_{1}$ & $2.2 \pm 0.1$ & $1.1 \pm 0.1$ & $6.5 \pm 0.1$ & $5.4 \pm 0.1$ & $0.6 \pm 0.1$ & $0.3 \pm 0.1$ & $15.7 \pm 7.8$ & $10.2 \pm 6.9$ \\
\hline $\mathrm{kT}_{2}$ & $6.1 \pm 0.1$ & $2.3 \pm 0.3$ & $6.8 \pm 0.1$ & $6.5 \pm 0.2$ & $6.8 \pm 0.1$ & $9.4 \pm 0.2$ & $7.6 \pm 0.7$ & $6.25 \pm 0.8$ \\
\hline$N_{2}$ & $1.8 \pm 0.1$ & $0.6 \pm 0.1$ & $8.9 \pm 0.1$ & $6.1 \pm 0.1$ & $0.8 \pm 0.1$ & $0.1 \pm 0.1$ & $44.0 \pm 6.8$ & $50.2 \pm 14.1$ \\
\hline $\mathrm{kT}_{3}$ & $7.3 \pm 0.2$ & $18.7 \pm 0.2$ & $10.7 \pm 0.2$ & $15.4 \pm 0.6$ & $10.3 \pm 0.3$ & $4.4 \pm 0.7$ & - & - \\
\hline$N_{3}$ & $1.6 \pm 0.1$ & $0.4 \pm 0.1$ & $8.9 \pm 0.1$ & $7.2 \pm 0.2$ & $0.7 \pm 0.1$ & $0.1 \pm 0.02$ & - & - \\
\hline $\mathrm{kT}_{4}$ & $10.9 \pm 0.5$ & $6.9 \pm 0.1$ & $7.4 \pm 0.2$ & $4.0 \pm 0.2$ & $6.9 \pm 0.2$ & - & - & - \\
\hline$N_{4}$ & $0.9 \pm 0.1$ & $1.0 \pm 0.1$ & $6.9 \pm 0.1$ & $4.6 \pm 0.2$ & $0.6 \pm 0.1$ & - & - & - \\
\hline Flux of S XVI & $7.9 \pm 0.1$ & $3.9 \pm 0.1$ & $39.1 \pm 6.6$ & $13.1 \pm 0.9$ & $2.9 \pm 0.1$ & $2.8 \pm 0.1$ & $49.1 \pm 7.3$ & $55.5 \pm 4.9$ \\
\hline Flux of Ca XIX & $2.4 \pm 0.1$ & $0.9 \pm 0.2$ & $13.5 \pm 4.8$ & $4.6 \pm 0.6$ & $0.7 \pm 0.1$ & $0.6 \pm 0.1$ & $25.6 \pm 1.5$ & $11.9 \pm 2.9$ \\
\hline Flux of $\mathrm{Ca} \mathrm{xx}$ & $1.7 \pm 0.1$ & $0.4 \pm 0.2$ & $8.5 \pm 0.5$ & $1.8 \pm 0.6$ & $0.5 \pm 0.1$ & $0.4 \pm 0.1$ & $14.7 \pm 1.2$ & $11.1 \pm 7.3$ \\
\hline
\end{tabular}
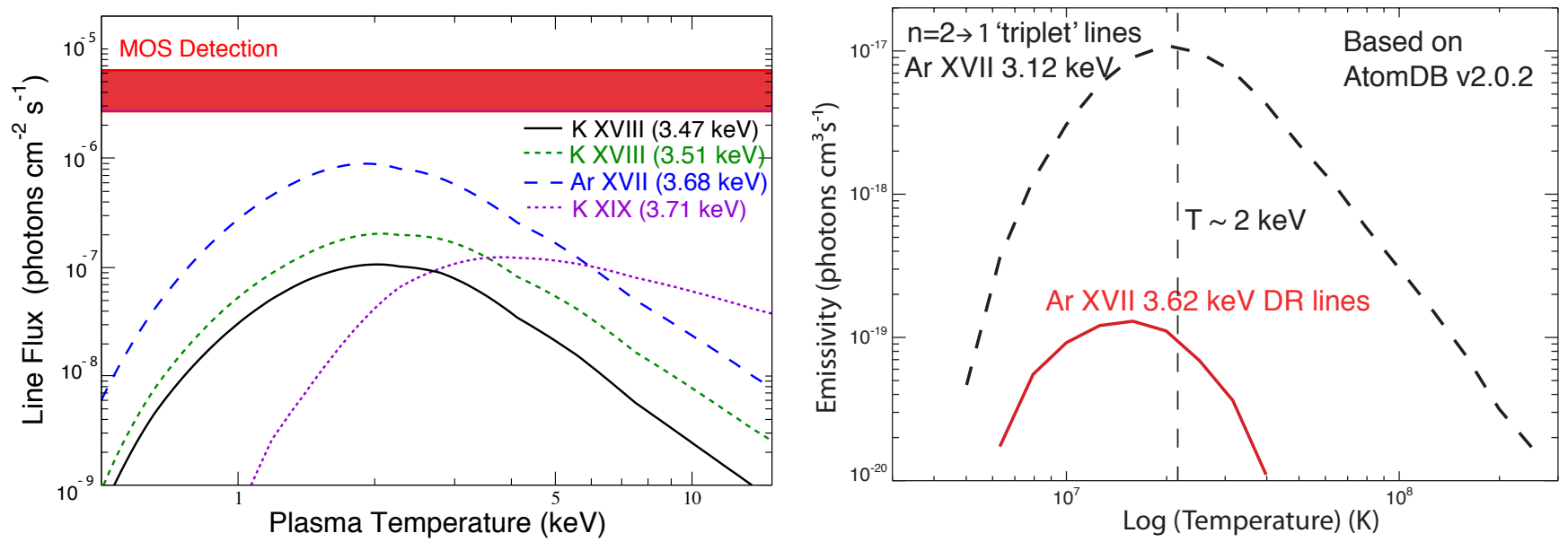

Figure 4. Left Panel: Eetimated line fluxes of the K XVIII at the rest energies $3.47 \mathrm{keV}, 3.51 \mathrm{keV}$, the Ar XVII at the rest energy $3.68 \mathrm{keV}$, and the $\mathrm{K}$ XIX at the rest energy $3.71 \mathrm{keV}$ as a function of plasma temperature. The line fluxes are calculated based on the observed fluxes of S XVI, Ca XIX, and Ca Xx from the stacked XMM-Newton MOS observations of the full sample. The flux detection and $90 \%$ errors on the flux of the unknown spectral feature measured from the stacked MOS observations of the full sample are shown with the red shaded area. Right Panel: Comparison of emissivities of the Ar XVII triplet lines at $3.12 \mathrm{keV}$ and Ar XVII DR line at $3.62 \mathrm{keV}$. The figure shows that the ratio of the Ar XVII DR line at $3.62 \mathrm{keV}$ to the Ar XVII line at $3.12 \mathrm{keV}$ could at most be $1 \%$ at the lowest temperature we observe in our fits $(T \sim 2 \mathrm{keV}$ indicated with the dashed line). This fraction was used as an upper limit to the flux of the Ar XVII DR line in our spectral fits and given in Table 3 for each sample.

the lines from AtomDB, we estimated the flux as

$$
\Gamma_{l}=\Gamma_{r} \sum^{i} \operatorname{Norm}_{i} \varepsilon_{l}\left(\mathrm{~T}_{e}\right) / \varepsilon_{r}\left(\mathrm{~T}_{e}\right)
$$

where subscripts $l$ and $r$ represent the lines of interest (K XVIII and Ar XVII) and reference lines (S XVI, Ca XIX, and $\mathrm{Ca} \mathrm{XX})$, respectively, $\Gamma$ is the flux in the line, $\varepsilon\left(T_{e}\right)$ is the calculated emissivity from AtomDB at the electron temperature $T_{e}$, and the sum over $i$ represents the different temperature components listed in Table 2 with their normalizations Norm $_{i}$. We use 0.1 and 3 times the maximum values of these fluxes as lower and upper bounds, respectively, for the normalizations of the Gaussian lines in the XSPEC fitting. The lower limits of 0.1 is set to avoid the lines vanishing and posing problems for the minimization routine. The factor three represents a conservative allowance for variation of the relative el- 
Table 3

Estimated Maximum Fluxes of K XVIII at the Rest Energies $3.47 \mathrm{keV}, 3.51 \mathrm{keV}$, Ar XVII at the Rest Energies $3.68 \mathrm{keV}$, and K XIX at the Rest energy $3.71 \mathrm{keV}$ Lines Obtained from AtomDB in the Units of photons $\mathrm{cm}^{-2} \mathrm{~s}^{-1}$. Estimates were performed based on best-fit fluxes obtained from the fluxes of S XVI, Ca XIX, and Ca Xx lines in the line-free apec model. The maximum flux for the Ar XVII DR line at 3.62 $\mathrm{keV}$ line was initially set to $1 \%$ of the Ar xvii line at 3.12. The fits were allowed to go a factor of 3 three above these estimates."

\begin{tabular}{|c|c|c|c|c|c|c|}
\hline Sample & Inst. & $\begin{array}{c}\text { Flux } \\
\text { K XVIII } \\
(3.47 \mathrm{keV}) \\
\left(10^{-7}\right)\end{array}$ & $\begin{array}{c}\text { Flux } \\
\text { K xviII } \\
(3.51 \mathrm{keV}) \\
\left(10^{-7}\right)\end{array}$ & $\begin{array}{c}\text { Flux } \\
\text { Ar XVII } \\
(3.62 \mathrm{keV}) \\
\left(10^{-7}\right)\end{array}$ & $\begin{array}{c}\text { Flux } \\
\text { Ar XVII } \\
(3.68 \mathrm{keV}) \\
\left(10^{-6}\right)\end{array}$ & $\begin{array}{c}\text { Flux } \\
\text { K XIX } \\
(3.71 \mathrm{keV}) \\
\left(10^{-6}\right)\end{array}$ \\
\hline \multirow{2}{*}{$\begin{array}{c}\text { Full } \\
\text { Sample }\end{array}$} & MOS & $1.3 \pm 0.7$ & $3.5 \pm 1.8$ & 0.12 & $1.0 \pm 0.5$ & $1.2 \pm 0.6$ \\
\hline & PN & $0.9 \pm 0.4$ & $1.8 \pm 0.9$ & 0.14 & $0.7 \pm 0.3$ & $0.3 \pm 0.1$ \\
\hline \multirow{2}{*}{$\begin{array}{c}\text { Coma }+ \\
\text { Centaurus }+ \\
\text { Ophiuchus }\end{array}$} & MOS & $2.7 \pm 2.1$ & $8.2 \pm 6.3$ & 7.0 & $2.5 \pm 1.9$ & $5.2 \pm 4.1$ \\
\hline & PN & $3.3 \pm 2.3$ & $6.8 \pm 4.7$ & 1.4 & $2.5 \pm 1.8$ & $0.8 \pm 0.6$ \\
\hline \multirow[t]{2}{*}{ Perseus } & MOS & $18.5 \pm 9.9$ & $45.7 \pm 24.4$ & 6.4 & $15.1 \pm 8.1$ & $11.6 \pm 6.2$ \\
\hline & $\mathrm{PN}$ & $13.8 \pm 6.8$ & $36.0 \pm 17.8$ & 1.99 & $10.8 \pm 5.4$ & $9.15 \pm 4.5$ \\
\hline $\begin{array}{c}\text { All } \\
\text { Other }\end{array}$ & MOS & $0.5 \pm 0.2$ & $1.3 \pm 0.5$ & 0.10 & $0.4 \pm 0.1$ & $0.29 \pm 0.1$ \\
\hline Clusters & $\mathrm{PN}$ & $1.3 \pm 0.5$ & $2.6 \pm 0.9$ & 0.90 & $1.1 \pm 0.4$ & $1.2 \pm 0.4$ \\
\hline
\end{tabular}

emental abundances between $\mathrm{S}$ and $\mathrm{Ca}$ (the measured lines on which the predictions are based) on one hand and $\mathrm{K}$ and $\mathrm{Ar}$ on the other. (This factor of three is not included in Table 3,

Since our detected emission line is only $50 \mathrm{eV}$ away from the Ar XVII DR at rest energy $3.62 \mathrm{keV}$, we calculated the emissivity of the Ar XVII DR line in a conservative way, using AtomDB v2.0.2. The He-like argon triplet including four lines (known either as $w, x, y, z$ or $R, I 1$, I2, and F) was summed, since the components cannot be distinguished at the CCD resolution. The two Ar XVII DR lines at $3.62 \mathrm{keV}$, known in AtomDB as $10077 \rightarrow 2$ and $10078 \rightarrow 3$, and which are the result of an He-like Ar ion recombining to Li-like $\mathrm{Ar}$ and emitting a photon at $3.62 \mathrm{keV}$, were similarly extracted and summed. The right panel of Figure 4 shows the comparison of the emissivity of Ar XVII DR and the He-like Argon triplet at $E \approx 3.12 \mathrm{keV}$. To model the flux of the Ar XVII DR line in our spectral fits in a conservative way, we set the lower and upper limits of the flux to be 0.001 and 0.01 times the flux of the He-like Ar, respectively. The upper limit corresponds to the highest flux that Ar XVII DR can have for the ICM plasma temperatures that we see in our spectra (this will be further discussed in Section 3.4). For a plasma temperature of $\sim 2 \mathrm{keV}$, the lowest temperature observed in our samples, the ratio of the flux of the Ar XVII DR line to He-like Ar line corresponds to 0.01 and thus was chosen as an upper limit (see Figure 4 right panel). The lower limit has been set to avoid problems with the fitting procedure.

Once the lower and upper limits on flux estimates of the K XVIII, KxIX, and Ar XVII lines were set, we performed the fit in a narrower $3-6 \mathrm{keV}$ energy band (to avoid strong $\mathrm{S}$ and $\mathrm{Si}$ lines below $3 \mathrm{keV}$ and $\mathrm{Fe}$ lines above $6 \mathrm{keV}$ ). This band is sufficiently wide to measure the continuum accurately (to better than $1 \%$ ). The weak residual emission line at $E \approx 3.57 \mathrm{keV}$ was detected in the fits. The excess emission after the Gaussian $\mathrm{K}$ and Ar lines were included in the model at their maximum fluxes (as described above) in MOS and PN spectra is shown in Figure 5. Figure 6 shows the excess in the rebinned MOS spectrum of the full sample. We then added a Gaussian model to fit the remaining residuals, leaving its flux and energy to vary. The fit was improved by $\Delta \chi^{2}$ of 22.8 for MOS and $\Delta \chi^{2}$ of 13.9 for PN for an additional 2 dof (energy and normalization). The best-fit energy of the added Gaussian line is $3.57 \pm 0.02(0.03) \mathrm{keV}$ in the stacked MOS and $3.51 \pm 0.03(0.04) \mathrm{keV}$ in the stacked $\mathrm{PN}$ observations. The line energies from MOS and PN are in significant tension, $2.8 \sigma$ apart (Figure 9). However, given the systematic uncertainties of the fitting procedure, we consider it acceptable; this tension disappears once another level of complexity is introduced in modeling (see Section 3.5 below). The width of the new line is unresolved and broadened only by the instrumental response. This is the only significant unidentified feature we have detected in the $2-10 \mathrm{keV}$ band of MOS and PN spectra.

To measure the flux of this line, we have to use a statistically proper response file, which will depend on the physical interpretation of the line. If the line were coming from the thermal plasma, then the same spectral responses that were used for the thermal components are appropriate. However, there are no known thermal plasma lines at this energy, so we explore a possible interpretation of the detected line as a decay signature of the sterile neutrino (see Section 1). In this interpretation, the spectral fitting procedure has to be slightly modified. 
In particular, when co-adding the instrumental responses used for the DM line component, the individual cluster responses should be weighted by the factor $\omega_{\mathrm{dm}}$ propor- tional to the estimated dark matter photon flux from each cluster (as opposed to the X-ray flux used for the response averaging so far).

Table 4 Columns (1) and (2) show the estimated projected dark matter masses in the spectral extraction radii $M_{D M}^{\text {proj }}$ ( $R_{\text {ext }}$ ) and the extraction radii $R_{\text {ext }}$ in Mpc, Column (3) is the projected dark matter masses per distance squared, and column (4) shows the ratio of the exposure time to the total exposure stacked for each cluster, column (5) is the weighting factors ( $\omega_{\text {dm }}$ ) calculated based on the predicted dark matter flux used in the stacking of ARFs and RMFs of each cluster in the sample. These stacked ARFs and RMFs were then used to determine the flux of the detected line, and column (6) shows the weighting factors ( $\omega_{\text {cnt }}$ ) calculated based on the total counts in the fitting band. The response files which were stacked using these factors were utilized to model plasma emission lines.

\begin{tabular}{|c|c|c|c|c|c|c|}
\hline \multirow[b]{2}{*}{ Cluster } & \multirow{2}{*}{$\begin{array}{c}\mathbf{( 1 )} \\
M_{D M}^{\text {proj }}\left(R_{\text {ext }}\right) \\
\left(10^{14} M_{\odot}\right) \\
\end{array}$} & \multirow{2}{*}{$\begin{array}{c}(\mathbf{2}) \\
R_{\text {ext }} \\
(\mathbf{M p c})\end{array}$} & $(3)$ & \multirow{2}{*}{$\begin{array}{c}(4) \\
\operatorname{Exp} / \operatorname{Exp}_{t o t}\end{array}$} & \multirow{2}{*}{$\begin{array}{c}\mathbf{5}) \\
\omega_{d m}\end{array}$} & \multirow{2}{*}{$\begin{array}{l}\mathbf{6}) \\
\omega_{c n t}\end{array}$} \\
\hline & & & $\begin{array}{c}M_{D M}^{\text {proj }} / \mathbf{D}^{2} \\
\left(10^{10} M_{\odot} / \mathbf{M p c}^{2}\right) \\
\end{array}$ & & & \\
\hline Centaurus & 0.63 & 0.17 & 2.41 & 0.044 & 0.139 & 0.074 \\
\hline A1060 & 0.59 & 0.21 & 1.82 & 0.010 & 0.024 & 0.009 \\
\hline $\mathrm{A} 262$ & 0.52 & 0.24 & 1.24 & 0.015 & 0.025 & 0.011 \\
\hline Perseus & 1.49 & 0.24 & 2.89 & 0.048 & 0.181 & 0.39 \\
\hline AWM7 & 0.86 & 0.24 & 1.82 & 0.045 & 0.106 & 0.061 \\
\hline Coma & 2.72 & 0.33 & 2.78 & 0.026 & 0.094 & 0.062 \\
\hline A3581 & 1.32 & 0.27 & 1.35 & 0.028 & 0.050 & 0.013 \\
\hline Ophiuchus & 4.14 & 0.38 & 3.05 & 0.009 & 0.037 & 0.032 \\
\hline A4038 & 1.31 & 0.39 & 0.91 & 0.008 & 0.010 & 0.007 \\
\hline A 496 & 2.29 & 0.44 & 1.24 & 0.038 & 0.061 & 0.044 \\
\hline A2063 & 1.92 & 0.48 & 0.88 & 0.008 & 0.009 & 0.0057 \\
\hline A 2147 & 2.06 & 0.47 & 0.96 & 0.002 & 0.003 & 0.0016 \\
\hline A3571 & 3.94 & 0.53 & 1.42 & 0.007 & 0.013 & 0.012 \\
\hline A3558 & 3.40 & 0.64 & 0.82 & 0.012 & 0.013 & 0.01 \\
\hline A4059 & 2.75 & 0.61 & 0.27 & 0.010 & 0.003 & 0.007 \\
\hline Triangulum Australis & 7.58 & 0.68 & 1.66 & 0.002 & 0.006 & 0.003 \\
\hline Hydra A & 2.68 & 0.72 & 0.51 & 0.025 & 0.016 & 0.023 \\
\hline A754 & 7.91 & 0.72 & 1.48 & 0.004 & 0.008 & 0.0032 \\
\hline A2319 & 6.93 & 0.72 & 1.31 & 0.003 & 0.0004 & 0.033 \\
\hline Cygnus A & 3.81 & 0.72 & 0.72 & 0.005 & 0.005 & 0.004 \\
\hline AS1101 & 1.95 & 0.79 & 0.34 & 0.025 & 0.011 & 0.0136 \\
\hline A3112 & 4.44 & 0.96 & 0.45 & 0.054 & 0.034 & 0.0337 \\
\hline A 2597 & 3.61 & 0.96 & 0.29 & 0.004 & 0.002 & 0.002 \\
\hline A478 & 8.30 & 1.10 & 0.61 & 0.019 & 0.014 & 0.017 \\
\hline PKS0745-19 & 10.03 & 1.27 & 0.52 & 0.005 & 0.003 & 0.003 \\
\hline A2811 & 5.29 & 1.08 & 0.15 & 0.007 & 0.001 & 0.0018 \\
\hline A2034 & 8.07 & 1.25 & 0.35 & 0.005 & 0.002 & 0.002 \\
\hline RXC J0616.8-4748 & 3.97 & 0.99 & 0.16 & 0.0069 & 0.001 & 0.0007 \\
\hline RXC J0145.0-5300 & 6.11 & 1.14 & 0.25 & 0.011 & 0.003 & 0.0025 \\
\hline RXC J1044.5-0704 & 3.05 & 0.89 & 0.09 & 0.007 & 0.0009 & 0.0014 \\
\hline A1068 & 4.44 & 1.01 & 0.12 & 0.005 & 0.0009 & 0.0012 \\
\hline RXC J2218.6-3853 & 6.68 & 1.16 & 0.20 & 0.005 & 0.001 & 0.0013 \\
\hline RXC J0605.8-3518 & 4.91 & 1.05 & 0.14 & 0.005 & 0.001 & 0.0013 \\
\hline A1413 & 9.09 & 1.29 & 0.24 & 0.053 & 0.016 & 0.018 \\
\hline A2204 & 8.86 & 1.27 & 0.21 & 0.019 & 0.005 & 0.010 \\
\hline A3888 & 8.57 & 1.26 & 0.20 & 0.013 & 0.003 & 0.004 \\
\hline RXC J0958.3-1103 & 6.58 & 1.15 & 0.15 & 0.002 & 0.0005 & 0.0006 \\
\hline A545 & 10.79 & 1.36 & 0.25 & 0.002 & 0.0005 & 0.0004 \\
\hline RXC J2014.8-2430 & 6.18 & 1.12 & 0.13 & 0.006 & 0.001 & 0.002 \\
\hline RX J1720.1+2638 & 6.64 & 1.25 & 0.13 & 0.016 & 0.003 & 0.004 \\
\hline RXC J0645.4-5413 & 8.55 & 1.47 & 0.16 & 0.005 & 0.001 & 0.001 \\
\hline A1201 & 5.78 & 1.10 & 0.11 & 0.015 & 0.002 & 0.0017 \\
\hline A1914 & 13.93 & 1.67 & 0.26 & 0.006 & 0.002 & 0.002 \\
\hline A 2345 & 7.65 & 1.20 & 0.14 & 0.015 & 0.003 & 0.002 \\
\hline A 2218 & 7.48 & 1.19 & 0.13 & 0.012 & 0.002 & 0.002 \\
\hline $\mathrm{A} 2254$ & 7.47 & 1.19 & 0.13 & 0.017 & 0.003 & 0.002 \\
\hline A665 & 9.50 & 1.29 & 0.16 & 0.006 & 0.001 & 0.0015 \\
\hline A1689 & 12.55 & 1.42 & 0.20 & 0.010 & 0.002 & 0.004 \\
\hline A383 & 3.48 & 0.92 & 0.05 & 0.008 & 0.0005 & 0.009 \\
\hline A520 & 7.75 & 1.20 & 0.11 & 0.009 & 0.001 & 0.001 \\
\hline
\end{tabular}


TABLE 4 - continued from previous page

\begin{tabular}{lcccccc}
\hline Cluster & $\begin{array}{c}M_{D M}^{\text {proj }}\left(R_{\text {ext }}\right) \\
\left(10^{14} M_{\odot}\right)\end{array}$ & $\begin{array}{c}R_{\text {ext }} \\
(\mathbf{M p c})\end{array}$ & $\begin{array}{c}M_{D M}^{\text {proj }} / \mathbf{D}^{2} \\
\left(10^{10} M_{\odot} / \mathbf{M p c}^{2}\right)\end{array}$ & $\mathbf{E x p / \mathbf { E x p } _ { \text { tot } }}$ & $\omega_{d m}$ & $\omega_{\text {cnt }}$ \\
\hline & & & & & & \\
A2163 & 26.34 & 1.80 & 0.34 & 0.003 & 0.001 & 0.001 \\
A209 & 8.82 & 1.25 & 0.11 & 0.005 & 0.0007 & 0.0007 \\
A963 & 6.81 & 1.15 & 0.07 & 0.006 & 0.0006 & 0.001 \\
RXC J1504.1-0248 & 8.87 & 1.25 & 0.09 & 0.011 & 0.003 & 0.004 \\
MS 0735.6+7421 & 3.89 & 0.95 & 0.04 & 0.014 & 0.0008 & 0.001 \\
A773 & 9.34 & 1.27 & 0.11 & 0.004 & 0.0005 & 0.0004 \\
AS0592 & 13.27 & 1.42 & 0.14 & 0.008 & 0.002 & 0.0017 \\
A2390 & 12.07 & 1.38 & 0.13 & 0.003 & 0.0005 & 0.0008 \\
A2667 & 9.66 & 1.28 & 0.10 & 0.006 & 0.0007 & 0.0011 \\
A267 & 4.83 & 1.01 & 0.05 & 0.002 & 0.0001 & 0.0005 \\
RXC J2129.6+0005 & 3.06 & 0.87 & 0.03 & 0.0097 & 0.0004 & 0.001 \\
RXC J1314.4-2515 & 8.61 & 1.22 & 0.07 & 0.010 & 0.0009 & 0.004 \\
A1835 & 12.15 & 1.37 & 0.10 & 0.037 & 0.005 & 0.009 \\
A1758 & 4.54 & 1.04 & 0.03 & 0.009 & 0.0004 & 0.0008 \\
A1763 & 10.47 & 1.32 & 0.11 & 0.004 & 0.0005 & 0.0005 \\
A689 & 22.51 & 1.66 & 0.15 & 0.002 & 0.0001 & 0.0001 \\
ZW 3146 & 6.72 & 1.11 & 0.04 & 0.059 & 0.003 & 0.010 \\
A781 & 5.57 & 1.04 & 0.03 & 0.018 & 0.0007 & 0.001 \\
Bullet & 15.24 & 1.45 & 0.09 & 0.006 & 0.0007 & 0.001 \\
MS 2137.3-2353 & 4.31 & 0.95 & 0.02 & 0.003 & 0.0001 & 0.0002 \\
MACS J2229.7-2755 & 3.51 & 0.88 & 0.02 & 0.009 & 0.0001 & 0.0006 \\
MACS J1532.8+3021 & 4.85 & 0.97 & 0.02 & 0.003 & 0.0007 & 0.0003 \\
AS1063 & 16.80 & 1.48 & 0.07 & 0.004 & 0.0004 & 0.0008 \\
& & & & & \\
\hline \hline
\end{tabular}

These response files will be solely used to measure the flux of the detected $3.57 \mathrm{keV}$ line; for the rest of the components, clearly originating in the ICM, the X-ray flux weighting is correct. The dark matter response weighting was done using the following approach.

The surface brightness of the DM decay signal is proportional to the DM column density $S_{D M}=$ $\int_{\text {l.o.s. }} \rho_{D M}(r) d r$. The observed photon flux from the DM decay into a solid angle $\Omega_{F O V}$ is given by

$$
F_{D M}=\frac{M_{D M}^{F O V}}{4 \pi D_{L}^{2}} \frac{\Gamma_{\gamma}}{m_{s}}(1+z) \text { photons } \mathrm{cm}^{-2} \mathrm{~s}^{-1} .
$$

where $\Gamma_{\gamma}$ and $m_{s}$ are the decay rate and mass of the sterile neutrino (see Equation 1 and Pal \& Wolfenstein (1982)), $M_{D M}^{F O V}$ is the projected DM mass within the spectral extraction region $\left(R_{e x t}\right.$, which is either $R_{500}$ or $\left.R_{F O V}\right)$, and $D_{L}$ is the luminosity distance.

The DM mass projected along the line of sight is

$$
M_{D M}^{F O V}=\int_{l o s} \rho_{D M}(r) d r
$$

where $\rho_{D M}(r)$ is the distribution of dark matter determined by the Navarro-Frenk-White (NFW) profile (Navarro et al. 1997) and given by

$$
\rho_{D M}(r)=\frac{\rho_{c}}{\left(r / r_{s}\right)\left(1+r / r_{s}\right)^{2}},
$$

where $\rho_{c}$ is a characteristic density and $r_{s}$ is a scale radius. The integration of the dark matter distribution within the extraction radius (given in Table 4) is along the line of sight. An extraction radius of $700^{\prime \prime}$ was used for the clusters larger than the field of view (FOV) of
XMM-Newton, while an extraction radius of $R_{500}$ was used for the clusters smaller than the FOV.

The expected contribution of each cluster $i$ to the total DM line flux in the stacked spectrum is

$$
\omega_{i, d m}=\frac{M_{i, D M}^{p r o j}\left(<R_{e x t}\right)\left(1+z_{i}\right)}{4 \pi D_{i, L}^{2}} \frac{e_{i}}{e_{t o t}} .
$$

where $z_{i}$ is the redshift of the $i$ th cluster, and $e_{i}$ and $e_{t o t}$ are the exposure time of the $i$ th cluster and the total exposure time of the sample.

The dark matter mass within the extraction radius is estimated as

$$
M_{D M}\left(R_{e x t}\right)=M_{t o t}\left(R_{e x t}\right)-M_{g a s}\left(R_{e x t}\right)-M_{*}\left(R_{e x t}\right),
$$

where $M_{t o t}\left(R_{e x t}\right), M_{g a s}\left(R_{e x t}\right)$, and $M_{*}\left(R_{e x t}\right)$ are the total mass, gas mass, and stellar mass in the extraction radius $R_{\text {ext }}$, respectively. The observed Vikhlinin et al. (2009) temperature-mass scaling relation was used to infer total masses for the intracluster gas temperatures measured from the XMM-Newton observations. The gas mass is determined following the method described in Bulbul et al. (2010). The contribution of stars to the total baryon budget is modest at large radii but more important in the cluster centers because of the presence of cD galaxies. At large radii $\left(\geq R_{500}\right), M_{*}$ is $10 \%-15 \%$ of the gas mass (Lin \& Mohr 2004, Vikhlinin et al. 2006). Stellar masses of each cluster were determined using the stellar mass - total mass scaling relation (Gonzalez et al. 2013). The calculated dark matter masses were corrected using this factor. The projected dark matter masses within $R_{\text {ext }}$ were then determined by projecting NFW profiles (Bartelmann 1996, Golse \& Kneib 2002, Loewenstein et al. 2009). We used a concentration parameter 
$c_{500}=3$ from the Vikhlinin et al. (2006) $c-M_{500}$ scaling relation and the median total mass within $R_{500}$ of the full sample, which is $\sim 6 \times 10^{14} M_{\odot}$. The projected dark matter mass within each spectral extraction radius is given in Table 4 .

Weights for the responses to be included in the stackedspectrum response were calculated as follows. The number of dark matter decay photons in each cluster spectrum is

$$
S_{i}=\alpha \omega_{i, d m} e_{t o t} A_{i},
$$

where $A_{i}$ is the ancillary response (the instrument effective area) at photon energy $E /\left(1+z_{i}\right)$, and $\alpha$ is the ratio of the decay rate of sterile neutrinos to the sterile neutrino mass $m_{s}$ (here we denote $\alpha \equiv \Gamma_{\gamma} / m_{s}$ ). The total number of dark matter photons in the stacked line is

$$
\begin{aligned}
S_{\text {line }} & =\sum_{i=0}^{i=73} S_{i} \\
& =\alpha \omega_{\text {tot }} e_{\text {tot }} A_{\omega},
\end{aligned}
$$

where the weighted ARF $A_{\omega}$ is a function of the total weight $\omega_{t o t}$,

$$
A_{\omega}=\sum_{i} \frac{\omega_{i}}{\omega_{t o t}} A_{i}
$$

and

$$
\omega_{t o t}=\sum_{i} \omega_{i} .
$$

The weighted responses $A_{\omega}$ were used to model our new line, while X-ray count-weighted response files were used to model the other known emission lines and the continuum components.

For MOS, the flux in the $3.57 \mathrm{keV}$ line was $4.0_{-0.8}^{+0.8}$ $\left(\begin{array}{c}+1.8 \\ -1.2\end{array}\right) \times 10^{-6}$ photons $\mathrm{cm}^{-2} \mathrm{~s}^{-1}$, where the errors are $68 \%(90 \%)$. For PN, at the best-fit energy of $3.51 \mathrm{keV}$, the line flux is $3.9_{-1.0}^{+0.6}\left({ }_{-1.6}^{+1.0}\right) \times 10^{-6}$ photons $\mathrm{cm}^{-2} \mathrm{~s}^{-1}$. If we fix the line energy from the MOS fit, for $\mathrm{PN}$ we obtain the flux $2.5_{-0.7}^{+0.6}\left({ }_{-1.1}^{+1.0}\right) \times 10^{-6}$ photons $\mathrm{cm}^{-2} \mathrm{~s}^{-1}$.

We note that the line energy detected in the stacked $\mathrm{PN}$ observations of the full sample is consistent with the $\mathrm{K}$ XVIII line at $3.515 \mathrm{keV}$. However, the measured flux from this line is a factor of 20 above the expected flux of the KXVIII line,estimated based on the measured fluxes of the $\mathrm{S}$ XVI, Ca XIX, and $\mathrm{Ca}$ XX lines and assuming a consistent relative abundance for KXVIII along with the plasma temperature from AtomDB. In addition, the detected energy in the stacked MOS observations of the full sample is $3.5 \sigma$ away from the $\mathrm{K}$ XVIII line. This will be further discussed later.

Since this is a blind search, in which the line energy is not known a priori, to estimate the significance of our detection, we must take into account the "look elsewhere" effect. We have examined $\sim 70$ independent energy resolution elements in our search band, and would accept a line detection in any of these bins. Taking this into account, our $4 \sigma-5 \sigma$ detections correspond to the probability of falsely detecting a line at an unknown energy of $0.004 \%$ for MOS and $0.4 \%$ for PN. However, the line is found at a consistent energy (or at least in the same independent resolution element) in these two completely independent samples coming from different instruments.
The statistical chance of such a false detection at the same energy is negligibly low. We caution that these are just the rough estimates of the statistical probabilities; systematic uncertainties are also important (Section 6).

Because estimating statistical significance of faint line features is a notoriously ill-behaved problem, we have verified the above estimate with a Monte Carlo simulations. We used the PN detection for this test, because its significance is lower and a Monte Carlo estimate can be done using a reasonable number of trials. We generated 1000 random realizations of a spectrum using a model in $X S P E C$ with no extra emission line and fit each of them with a model that included an additional line at an arbitrary location and flux. We then counted the realizations in which the model with the additional line improved the fit by $\Delta \chi>11.2$, which corresponds to our PN detection. This false detection occurred in 4 cases out of 1000 , in agreement with the above $0.4 \%$ probability of false detection in the stacked PN spectrum.

We also fit the same MOS and PN spectra using the X-ray count-weighted responses, to check if the detection is dependent on the response weighting. For MOS, the flux of the detected line was $4.1_{-0.9}^{+1.0}\left({ }_{-1.6}^{+1.8}\right)$ $\times 10^{-6}$ photons $\mathrm{cm}^{-2} \mathrm{~s}^{-1}$; the fit was improved by $\Delta \chi^{2}$ of 21.8 for 2 dof. For PN, the line flux was $3.9_{-1.0}^{+1.3}$ $\left(\begin{array}{c}+2.1 \\ -2.0\end{array}\right) \times 10^{-6}$ photons $\mathrm{cm}^{-2} \mathrm{~s}^{-1}$, while the fit was improved by $\Delta \chi^{2}$ of 13.8 for 2 dof. This shows that the detection is robust and the flux is independent of the response scaling.

We will discuss the possible physical interpretations of this emission line in Section 5. Here we will push forward with one possible interpretation of the detected line, sterile neutrino decay, because we need to describe the calculation of certain quantities that will be used below for cross-checks and comparison of the subsamples of our full sample.

For a DM particle decaying radiatively with $E_{\gamma}=$ $m_{s} / 2$, the detected flux from a clump of matter of a known mass can be converted into the decay rate. The energy of the detected line corresponds to a sterile neutrino particle mass of $m_{s}=7.1 \pm 0.07 \mathrm{keV}$, assuming that the dark matter is solely composed of sterile neutrinos. The relation between the flux and mass implies a mixing angle of

$$
\begin{array}{r}
\sin ^{2}(2 \theta)=\frac{\mathrm{F}_{\mathrm{DM}}}{12.76 \mathrm{~cm}^{-2} \mathrm{~s}^{-1}}\left(\frac{10^{14} \mathrm{M}_{\odot}}{\mathrm{M}_{\mathrm{DM}}^{\mathrm{FOV}}}\right) \\
\left(\frac{D_{L}}{100 \mathrm{Mpc}}\right)^{2}\left(\frac{1}{1+z}\right)\left(\frac{1 \mathrm{keV}}{m_{s}}\right)^{4}
\end{array}
$$

where $F_{D M}$ is the observed DM flux.

Using the $\omega_{d m}$ and the projected dark matter masses given in Table 4, we find that the weighted projected dark matter mass per distance squared is $1.82 \times 10^{10}$ $M_{\odot} / M p c^{2}$ for the full sample observed with XMMNewton MOS. Using Equation (3), one can calculate the mixing angle for the full MOS cluster sample to be $\sin ^{2}(2 \theta)=6.8_{-1.4}^{+1.4}\left({ }_{-3.0}^{+2.0}\right) \times 10^{-11}$. The PN observations of the full sample give a mixing angle measurement of $\sin ^{2}(2 \theta)=6.7_{-1.0}^{+1.7}\left({ }_{-1.7}^{+2.7}\right) \times 10^{-11}$ for a weighted mass per distance squared of $1.80 \times 10^{10} M_{\odot} / M p c^{2}$. These are given in Table 5. The PN and MOS full-sample measure- 

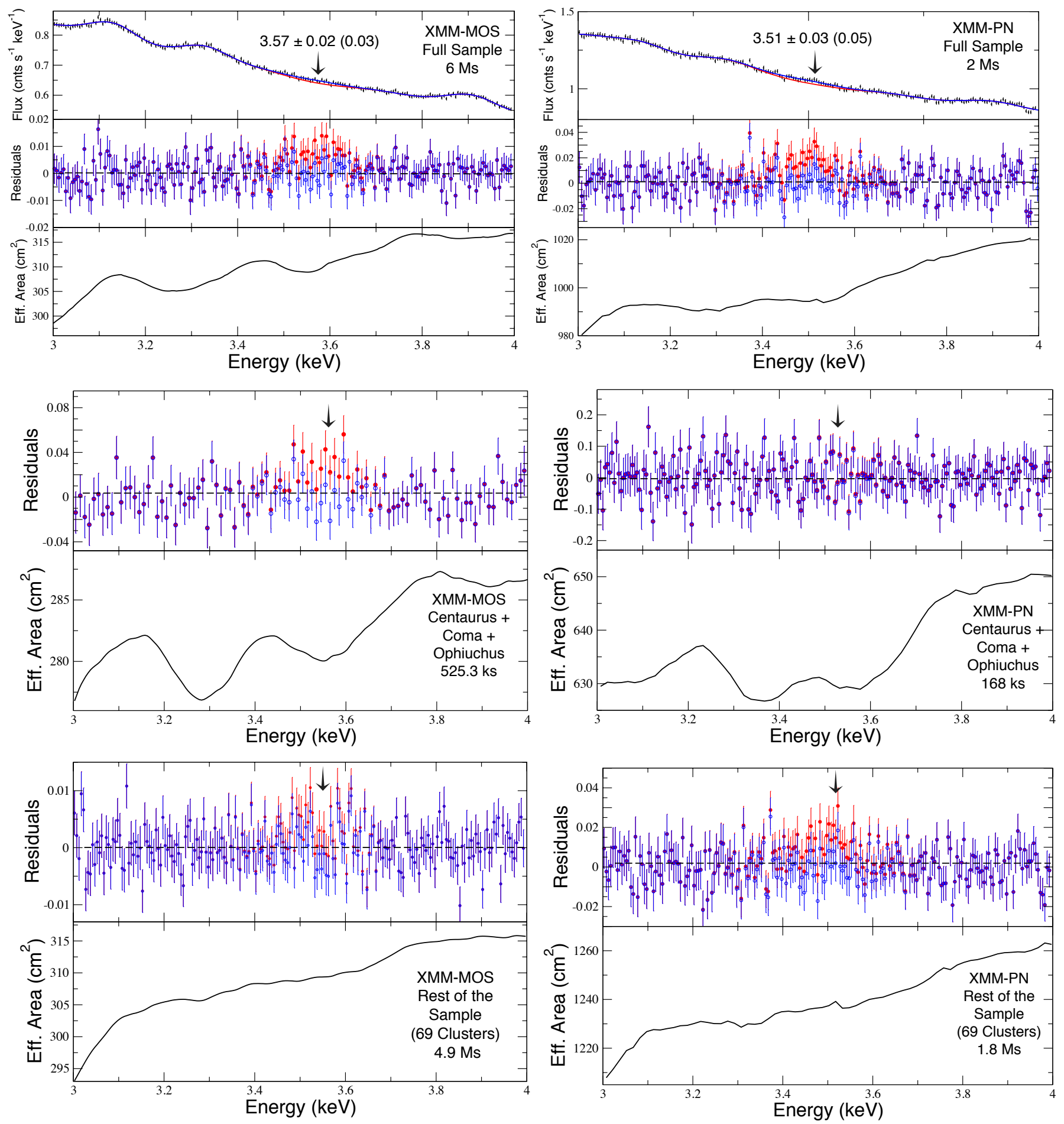

Figure 5. Top panels: $3-4 \mathrm{keV}$ band of the stacked MOS (left panel) and stacked PN (right panel) spectra of the samples. The figures show the energy band where the new spectral feature is detected. The Gaussian lines with maximum values of the flux normalizations of $\mathrm{K}$ XVIII and Ar XVII estimated using AtomDB were included in the models. The red lines in the top panels (shown only for the full sample) show the model and the excess emission. The blue lines show the total model after another Gaussian line is added, representing the new line. Middle panels show the residuals before (red) and after (blue) the Gaussian line is added. The bottom panels show the effective area curves (the corresponding ARF). Redshift smearing greatly reduces variations of the effective area in the high- $z$ sample.

ments are consistent with each other and the constraints placed by previous studies, e.g., the unresolved cosmic X-ray background (CXB) in the Chandra Deep Fields (Abazajian et al. 2007) and the XMM-Newton blanksky background spectrum (Boyarsky et al. 2006), Chan- dra observations of the Milky Way (Riemer-Sørensen et al. 2006), Chandra observation of the Bullet Cluster (Boyarsky et al. 2008), Chandra observations of the dwarf galaxy Draco (Riemer-Sørensen \& Hansen 2009), and XMM-Newton limits from M31 and Willman 1 and For- 

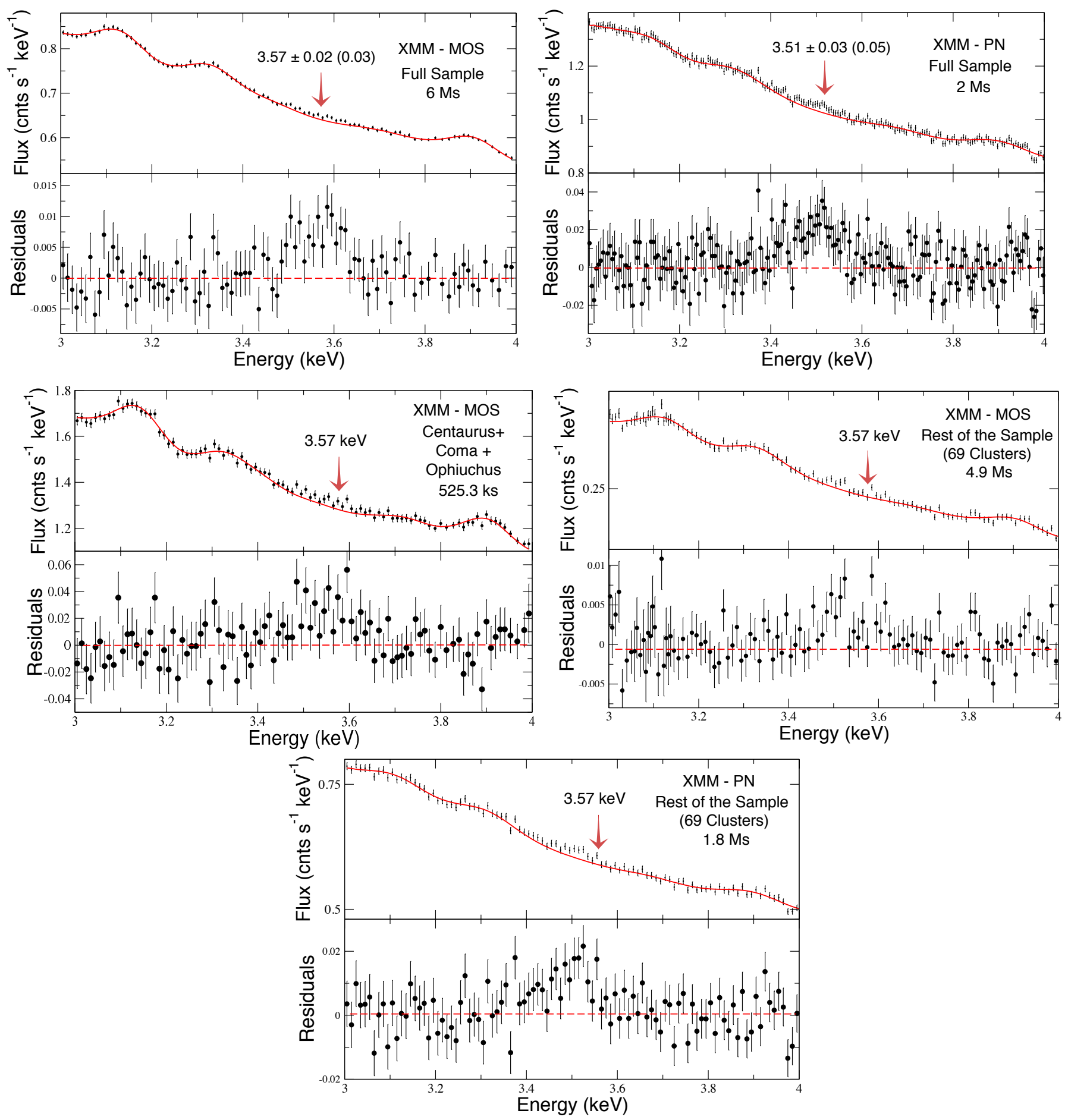

Figure 6. $3-4 \mathrm{keV}$ band of the rebinned XMM-Newton spectra of the detections. The spectra were rebinned to make the excess at $\sim 3.57$ keV more apparent. (APJ VERSION INCLUDES ONLY THE REBINNED MOS SPECTRUM OF THE FULL SAMPLE).

nax dwarf galaxies (Boyarsky et al. 2010: Watson et al. 2012), as showin in Figure 13(a). It is in marginal ( $\sim 90 \%$ significance) tension with the most recent Chandra limit from M31 (Horiuchi et al. 2014), as shown in Figure $13(\mathrm{~b})$.

For the PN flux for the line fixed at the best-fit MOS energy, the corresponding mixing angle is $\sin ^{2}(2 \theta)=$ $4.3_{-1.0}^{+1.2}\left({ }_{-1.7}^{+1.8}\right) \times 10^{-11}$. This measurement is consistent with that obtained from the stacked MOS observations at a $1 \sigma$ level. Since the most confident measurements are provided by the highest signal-to-noise ratio stacked MOS observations of the full sample, we will use the flux at energy $3.57 \mathrm{keV}$ when comparing the mixing angle measurements for the sterile neutrino interpretation of this line.

\subsection{Excluding Bright Nearby Clusters from the Sample}


Table 5

Columns (2) and (3) are the measured rest energy and flux of the unidentified line in the units of photons $\mathrm{cm}^{-2} \mathrm{~s}^{-1}$ at the $68 \%(90 \%)$ confidence level. The energy's with asterisks are frozen to the indicated values; column (4) and (5) show the $\chi^{2}$ before the line is added to

the total model and change in the $\chi^{2}$ when an additional Gaussian component is added to the fit; column (6) is the weighted ratio of mass to distance squared of the samples, and column (7) shows the mixing angle limits measured in each sample. Reported constraining limits are $90 \%$ confidence upper limits. Energies marked with star symbols were held fixed during the model fitting

\begin{tabular}{|c|c|c|c|c|c|c|c|c|}
\hline Sample & & $\begin{array}{l}(1) \\
\text { Inst. }\end{array}$ & $\begin{array}{c}(2) \\
\text { Energy } \\
(\mathrm{keV})\end{array}$ & $\begin{array}{c}(3) \\
\text { Flux } \\
\left(10^{-6} \text { phts cm }^{-2} \mathrm{~s}^{-1}\right)\end{array}$ & $\begin{array}{c}(4) \\
\chi^{2} \\
(\mathrm{dof})\end{array}$ & $\begin{array}{c}(5) \\
\Delta \chi^{2} \\
(\Delta \text { dof })\end{array}$ & $\begin{array}{c}(6) \\
\mathrm{M}_{D M}^{\text {proj }} / \mathrm{D}^{2} \\
\left(10^{10} \mathrm{M}_{\odot} / \mathrm{Mpc}^{2}\right)\end{array}$ & $\begin{array}{c}(7) \\
\sin ^{2}(2 \theta) \\
\left(10^{-11}\right)\end{array}$ \\
\hline \multirow{3}{*}{$\begin{array}{c}\text { Full } \\
\text { Sample }\end{array}$} & \multirow{3}{*}{$X M M$} & MOS & $3.57 \pm 0.02(0.03)$ & $4.0_{-0.8}^{+0.8}\left({ }_{-1.2}^{+1.8}\right)$ & $\begin{array}{l}564.8 \\
(566)\end{array}$ & $\begin{array}{c}22.8 \\
(2)\end{array}$ & 1.82 & $6.8_{-1.4}^{+1.4}\left({ }_{-3.0}^{+2.0}\right)$ \\
\hline & & $\mathrm{PN}$ & $3.51 \pm 0.03(0.05)$ & $3.9_{-1.0}^{+0.6}\left({ }_{-1.6}^{+1.0}\right)$ & $\begin{array}{l}510.5 \\
(564)\end{array}$ & $\begin{array}{l}13.9 \\
(2)\end{array}$ & 1.80 & $6.7_{-1.0}^{+1.7}\left({ }_{-1.7}^{+2.7}\right)$ \\
\hline & & $\mathrm{PN}$ & $3.57^{\star}$ & $2.5_{-0.7}^{+0.6}\left({ }_{-1.1}^{+1.0}\right)$ & $\begin{array}{l}510.5 \\
(564)\end{array}$ & $\begin{array}{c}11.2 \\
(1)\end{array}$ & 1.80 & $4.3_{-1.0}^{+1.2}\left({ }_{-1.7}^{+1.8}\right)$ \\
\hline \multirow{2}{*}{$\begin{array}{l}\text { Coma }+ \\
\text { Centaurus }+ \\
\text { Ophiuchus }\end{array}$} & \multirow{2}{*}{$X M M$} & MOS & $3.57^{\star}$ & $15.9_{-3.8}^{+3.4}\left(\begin{array}{l}+6.7 \\
-5.5\end{array}\right)$ & $\begin{array}{l}562.3 \\
(569)\end{array}$ & $\begin{array}{c}17.1 \\
(1)\end{array}$ & 2.68 & $18.2_{-3.9}^{+4.4}\left({ }_{-11.5}^{+12.6}\right)$ \\
\hline & & PN & $3.57^{\star}$ & $<9.5$ & $\begin{array}{l}377.8 \\
(387)\end{array}$ & - & - & $<10.9$ \\
\hline \multirow{2}{*}{$\begin{array}{l}\text { Perseus } \\
\text { (without } \\
\text { the core) }\end{array}$} & \multirow{2}{*}{$X M M$} & MOS & $3.57^{\star}$ & $21.4_{-6.3}^{+7.0}\left({ }_{-10.5}^{+11.2}\right)$ & $\begin{array}{l}596.1 \\
(574)\end{array}$ & $\begin{array}{c}12.8 \\
(1)\end{array}$ & 2.82 & $23.3{ }_{-6.9}^{+7.6}\left({ }_{-11.5}^{+12.2}\right)$ \\
\hline & & PN & $3.57^{\star}$ & $<16.1$ & $\begin{array}{l}539.1 \\
(553)\end{array}$ & - & - & $<17.6$ \\
\hline \multirow{2}{*}{$\begin{array}{l}\text { Perseus } \\
\text { (with } \\
\text { the core) }\end{array}$} & \multirow{2}{*}{$X M M$} & MOS & $3.57^{\star}$ & $52.0{ }_{-15.2}^{+24.1}\left(\begin{array}{l}+37.0 \\
-21.3\end{array}\right)$ & $\begin{array}{l}613.8 \\
(574)\end{array}$ & $\begin{array}{c}15.7 \\
(1)\end{array}$ & 2.89 & $55.3_{-15.9}^{+25.5}\left(\begin{array}{l}+39.3 \\
-22.6\end{array}\right)$ \\
\hline & & $\mathrm{PN}$ & $3.57^{\star}$ & $<17.7$ & $\begin{array}{l}539.4 \\
(554)\end{array}$ & - & - & $<18.8$ \\
\hline \multirow{2}{*}{$\begin{array}{c}\text { All } \\
\text { Other } \\
\text { Clusters }\end{array}$} & \multirow{2}{*}{$X M M$} & MOS & $3.57^{\star}$ & $2.1_{-0.5}^{+0.4}\left({ }_{-0.8}^{+0.8}\right)$ & $\begin{array}{l}547.2 \\
(573)\end{array}$ & $\begin{array}{l}16.5 \\
(1)\end{array}$ & 1.08 & $6.0_{-1.4}^{+1.1}\left({ }_{-2.3}^{+2.3}\right)$ \\
\hline & & PN & $3.57^{\star}$ & $2.0{ }_{-0.5}^{+0.3}\left(\left(_{-0.8}^{+0.5}\right)\right.$ & $\begin{array}{l}741.9 \\
(751)\end{array}$ & $\begin{array}{c}15.8 \\
(1)\end{array}$ & 1.15 & $5.4_{-1.3}^{+0.8}\left({ }_{-2.1}^{+1.3}\right)$ \\
\hline \multirow{2}{*}{ Perseus } & \multirow{2}{*}{ Chandra } & ACIS-S & $3.56 \pm 0.02(0.03)$ & $10.2{ }_{-3.5}^{+3.7}\left(\begin{array}{l}+4.8 \\
-4.7\end{array}\right)$ & $\begin{array}{c}201 \\
(197)\end{array}$ & $\begin{array}{l}11.8 \\
(2)\end{array}$ & 0.72 & $40.1_{-13.7}^{+14.5}\left({ }_{-18.2}^{+18.9}\right)$ \\
\hline & & ACIS-I & $3.56^{\star}$ & $18.6{ }_{-8.0}^{+7.8}\left({ }_{-16.0}^{+12.0}\right)$ & $\begin{array}{l}152.6 \\
(151)\end{array}$ & $\begin{array}{l}6.2 \\
(1)\end{array}$ & 1.86 & $28.3_{-12.1}^{+11.8}\left(\begin{array}{l}+18.2 \\
-24.3\end{array}\right)$ \\
\hline Virgo & Chandra & ACIS-I & $3.56^{\star}$ & $<9.1$ & $\begin{array}{l}189.1 \\
(155)\end{array}$ & - & 2.41 & $<10.5$ \\
\hline
\end{tabular}

We now divide the full cluster sample into three independent subsamples, in order to check that our line does not originate from any single object. The full stacked spectra examined in Section 3.1 have a significant contribution of photons from several nearby bright clusters, e.g., Perseus, Coma, Centaurus, and Ophiuchus. In order to determine whether the line detection is dominated by these bright sources, we excluded them from the sample and stacked the MOS and PN spectra of the remaining 69 fainter galaxy clusters. We have performed the stacking process following the same approach described in Section 2.3. A total of 4.9 Ms of good stacked MOS and 
1.7 Ms good stacked PN exposure were obtained for this subsample. The weighted mean redshift was 0.06 . The stacked MOS and PN spectra contain 34\% $\left(2.95 \times 10^{6}\right.$ source counts) and $55 \%\left(2.79 \times 10^{6}\right.$ source counts $)$ of the total source counts of the full cluster sample.

We fit the stacked spectra using the line-free apec model and additional Gaussian models as described in Section 3.1 in the $3-6 \mathrm{keV}$ band. The best-fit temperatures, normalizations of the line-free apec model, and the fluxes of S XVI, Ca XIX, and Ca XX lines are given in Table 2. We then carefully examined the spectra for any unidentified emission features in the $3.4-3.7 \mathrm{keV}$ energy interval. Similarly, we determined the maximum fluxes of the K XVIII, K XIX, and Ar XVII lines based on the plasma temperatures and fluxes of hydrogen-like $\mathrm{S}$ XVI, helium-like Ca XIX, and hydrogen-like Ca Xx lines at $2.63 \mathrm{keV}, 3.90 \mathrm{keV}$, and $4.11 \mathrm{keV}$, measured from the spectral fits, and AtomDB as described in Section 3.1. As before, the lower and upper limits of the fluxes of $\mathrm{K}$ XVIII, K XIX, and Ar XVII lines were set to 0.1 to 3 times of the maximum predicted fluxes. The Ar XVII DR line flux at $3.62 \mathrm{keV}$ was allowed to vary between $10^{-3}$ and $10^{-2}$ of the Ar XVII triplet line at $3.12 \mathrm{keV}$.

We obtained an acceptable fit to the stacked MOS spectrum of these 69 clusters. The total $\chi^{2}$ was 557 for 573 degrees of freedom. Adding in an extra Gaussian model to the MOS spectrum at $3.57 \mathrm{keV}$ improved the fit by $\Delta \chi^{2}$ of 16.5 for an additional dof. We found that the best-fit flux was $2.1{ }_{-0.5}^{+0.4}\left({ }_{-0.8}^{+0.8}\right) \times 10^{-6}$ photons $\mathrm{cm}^{-2} \mathrm{~s}^{-1}$. This flux corresponds to a mixing angle of $\sin ^{2}(2 \theta)=6.0{ }_{-1.4}^{+1.1}\left({ }_{-2.3}^{+2.3}\right) \times 10^{-11}$, consistent with the mixing angle estimates obtained from the full sample.

The overall fit to the stacked PN spectrum for these 69 clusters was acceptable with a total $\chi^{2}$ of 741.9 for 751 dof. Adding an extra Gaussian line at $3.57 \mathrm{keV}$ improved the fit by $\Delta \chi^{2}$ of 15.8 for an additional degree of freedom. The PN spectrum yields the best-fit flux detection of $2.0_{-0.5}^{+0.3}\left({ }_{-0.8}^{+0.5}\right) \times 10^{-6}$ photons $\mathrm{cm}^{-2} \mathrm{~s}^{-1}$. The mixing angle obtained from the stacked PN observations $\sin ^{2}(2 \theta)=5.4_{-1.3}^{+0.8}\left({ }_{-2.1}^{+1.3}\right) \times 10^{-11}$ is also consistent with the estimates from the full sample. Bottom panels in Figure 5 show the residuals before and after a Gaussian line is added at $3.57 \mathrm{keV}$ to MOS and PN spectral fits.

\subsection{Stacked Spectra of the Nearby Bright Clusters; Centaurus + Coma + Ophiuchus}

We now check the MOS and PN spectra of the three dominant nearby clusters, Coma, Ophiuchus, and Centaurus. A total of $525.3 \mathrm{ks}$ of good stacked MOS and 168 ks good stacked PN exposure times were obtained for this subsample. The total source counts obtained in the MOS and PN spectra were $3.2 \times 10^{6}$ and $2.1 \times 10^{6}$, respectively.

We performed the fits as above. The best determinations for the continuum temperature and normalizations and the fluxes of the S XVI, Ca XIX, and Ca XX are given in Table 2. We detected an excess emission feature in the same band, i.e. $3.4-3.7 \mathrm{keV}$ as in the stacked MOS spectra. To determine the flux of the emission line at 3.57 $\mathrm{keV}$, we estimated the maximum fluxes of the K XVIII, K XIX, and Ar XVII lines using the AtomDB and the measured fluxes of S XVI, Ca XIX, and Ca XX as described in Section 3.1. Using 0.1 and 3 times these fluxes as lower and upper limits, we found that the unidentified line has a flux of $1.6_{-0.4}^{+0.3}\left({ }_{-0.6}^{+0.7}\right) \times 10^{-5}$ photons $\mathrm{cm}^{-2} \mathrm{~s}^{-1}$ in the stacked MOS observations. Adding this Gaussian to the model improves the fit by $\Delta \chi^{2}$ of 17.1 for an additional degree of freedom for the stacked MOS spectrum.

We then allowed the energy of the additional Gaussian model to vary to test whether the energy measured from two different samples is the same. The best-fit energy obtained from the stacked MOS observations of Coma, Centaurus, and Ophiuchus clusters was $3.56 \pm 0.02(0.03)$, with a flux of $1.6_{-0.44}^{+0.52}\left({ }_{-0.70}^{+0.81}\right) \times 10^{-5}$ photons $\mathrm{cm}^{-2} \mathrm{~s}^{-1}$. This measurement is consistent with the energy measured in the MOS observations of the full sample. The sterile neutrino mixing angle that corresponds to this flux is $\sin ^{2}(2 \theta)=18.2_{-3.9}^{+4.4}\left({ }_{-11.5}^{+12.6}\right) \times 10^{-11}$, consistent at $2 \sigma$ with the full-sample value.

The fits to the stacked PN observations did not need an additional Gaussian line, and resulted in a non-detection. This could be due to the low count statistics of the stacked PN observations (168 ks clean time). A $90 \%$ upper limit on the flux of this line at $3.57 \mathrm{keV}$ is 9.5 $\times 10^{-6}$ photons $\mathrm{cm}^{-2} \mathrm{~s}^{-1}$ from this spectrum; the upper limit on the mixing angle from this flux limit is consistent with the full-sample and MOS detections.

\subsection{Perseus}

Initially, we extracted the spectrum of the Perseus Cluster using the entire MOS and PN FOV. We have co-added the XMM-Newton MOS and PN observations of the Perseus Cluster in the cluster's frame. The total exposure time in the stacked MOS spectrum was $317 \mathrm{ks}$ with a total of $7 \times 10^{6}$ source counts in the $2-10 \mathrm{keV}$ band and 38 ks total exposure with $2 \times 10^{6}$ source counts in the stacked PN observations.

Following the same approach we used for modeling the full cluster sample, we first fit the MOS and PN observations with the line-free apec model and additional Gaussian models. Count-weighted responses were used to fit the plasma emission lines and the continuum emission. Probing the 3-4 keV band the MOS observations revealed residuals around $3.57 \mathrm{keV}$, at the same energy band where we detected line emission in the previous samples. The left panel of Figure 7 shows the detection in the co-added MOS observations of the Perseus Cluster. Using the limits on the $\mathrm{K}$ and Ar lines (Table 3) as above and adding a Gaussian model to the MOS spectrum at the fixed energy of $3.57 \mathrm{keV}$ improved the fit by $\Delta \chi^{2}$ of 15.7. The best-fit flux at $3.57 \mathrm{keV}$ was $5.2_{-1.5}^{+2.4}$ $\left({ }_{-2.1}^{+3.7}\right) \times 10^{-5}$ photons $\mathrm{cm}^{-2} \mathrm{~s}^{-1}$.

This flux corresponds to a mixing angle of $\sin ^{2}(2 \theta)=$ $5.5_{-1.6}^{+2.6}\left({ }_{-2.3}^{+3.9}\right) \times 10^{-10}$. This angle not only is an outlier in our measurements from the other samples, but is also not consistent with the upper limits on the mixing angle at this value of $m_{s}$ from the previous studies (e.g., Horiuchi et al. 2014).

We were unable to detect the line in the short $(38 \mathrm{ks}$ clean time) PN observation of Perseus and placed a $90 \%$ upper limit on the flux of the line of 17.7 photons $\mathrm{cm}^{-2}$ $\mathrm{s}^{-1}$, which corresponds to an upper limit of $\sin ^{2}(2 \theta)<$ $1.9 \times 10^{-10}$, consistent with the MOS detection. Figure 7 shows both XMM-Newton Perseus spectra.

Since this is a single-cluster spectrum, we first check 

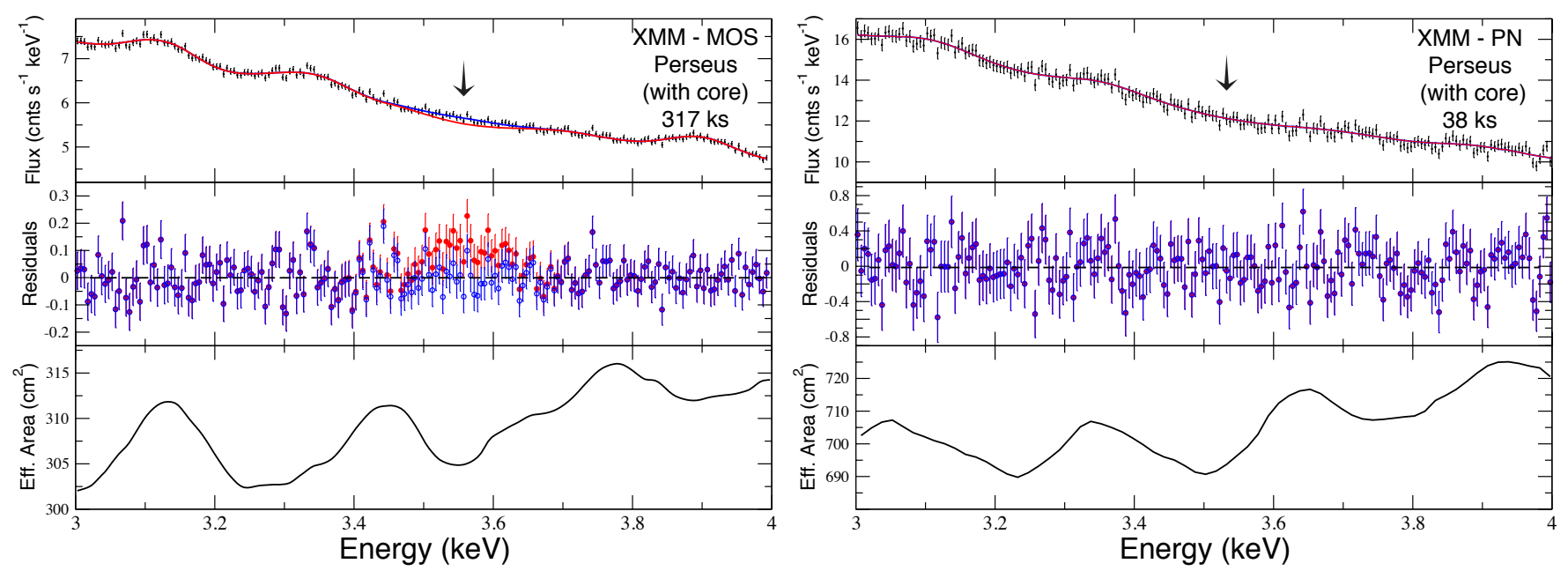

Figure 7. 3-4 keV band of the stacked MOS (left panel) and stacked PN (right panel) spectra of the Perseus Cluster. The figures show the energy band, where a new spectral feature at $3.57 \mathrm{keV}$ is detected. The Gaussian lines with peak values of the flux normalizations of K XVIII and Ar XVII estimated using AtomDB were included in the models. The red lines in the top panels show the model and the excess emission in both spectra. The blue lines show the total model after a Gaussian line is added, indicating that the unidentified spectral line can be modeled with a Gaussian.

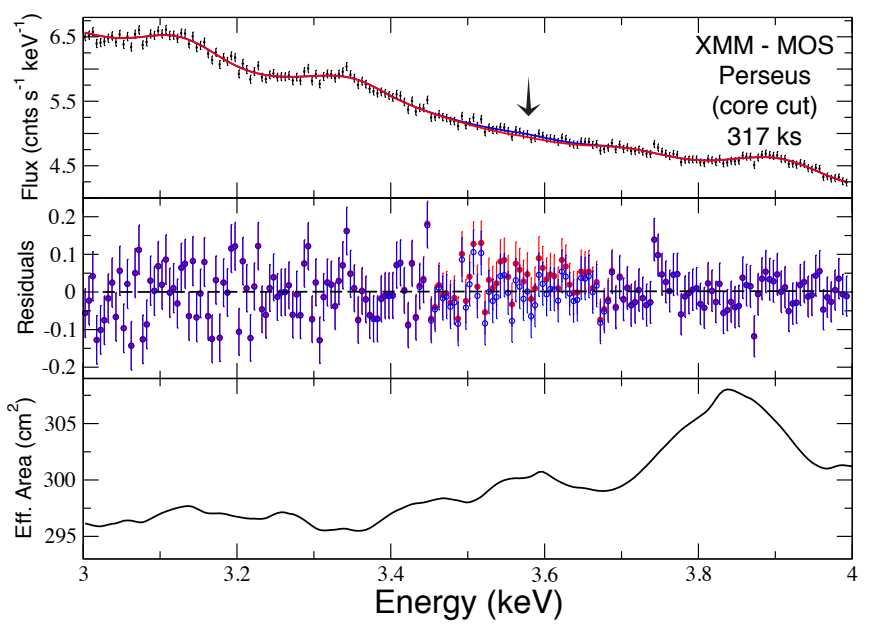

Figure 8. $3-4 \mathrm{keV}$ band of the core-excised stacked MOS spectrum of the Perseus Cluster. The figures show the energy band, where a new spectral feature at $3.57 \mathrm{keV}$ is detected. The Gaussian lines with peak values of the flux normalizations of K XVIII and Ar XVII estimated using AtomDB were included in the models. The red lines in the top panels show the model and the excess emission in both spectra. The blue lines show the total model after a Gaussian line is added, indicating that the unidentified spectral line can be modeled with a Gaussian.

whether the Perseus signal is not an artifact of our blueshifting procedure. For this we fit the original, redshifted MOS spectrum with a line-free apec model. We obtained a best-fit $\chi^{2}$ of 463 for 385 dof. Adding a Gaussian line at $3.57 \mathrm{keV}$ (rest energy) improved the fit by $\Delta \chi^{2}$ of 16 for an additional dof. The best-fit flux was $5.3 \pm 1.2(2.0) \times 10^{-5}$ photons $\mathrm{cm}^{-2} \mathrm{~s}^{-1}$, which is in agreement with the flux obtained from the blue-shifted spectrum. We conclude that our detection is independent of shifting the spectrum.

Not ready to abandon the sterile neutrino explanation based on the line flux incorrectly scaling with cluster mass that we see for Perseus, we tried to investigate possible astrophysical reasons behind the excess of the line flux in Perseus. First, we investigated the dependence of the energy and flux of this unidentified line on the AtomDB predicted fluxes of nearby lines, i.e., the $\mathrm{K}$ XVIII line at $3.51 \mathrm{keV}$ and the Ar XVII DR line at 3.62 $\mathrm{keV}$. Allowing the energy of the Gaussian component to vary produced a best fit for an energy of $3.566_{-0.02}^{+0.01}\left({ }_{-0.03}^{+0.02}\right)$ $\mathrm{keV}$, with a flux of $6.0_{-1.4}^{+1.8}\left({ }_{-1.7}^{+2.4}\right) \times 10^{-5}$ photons $\mathrm{cm}^{-2}$ $\mathrm{s}^{-1}$ ( $\chi^{2}$ of 598.1 for 572 dof). The best-fit energy is consistent with the energy measured from the MOS observations of the full sample. However, the fluxes of the nearby $\mathrm{K}$ XVIII line at $3.51 \mathrm{keV}$ and the Ar XVII DR line at $3.62 \mathrm{keV}$ were at their allowed upper limits predicted from AtomDB. Relaxing the upper limits has shifted the line energy higher, to $3.59_{-0.03}^{+0.01}\left({ }_{-0.04}^{+0.02}\right) \mathrm{keV}$ with a flux of $5.5_{-0.8}^{+1.7}\left({ }_{-1.5}^{+3.7}\right) \times 10^{-5}$ photons $\mathrm{cm}^{-2} \mathrm{~s}^{-1}$ giving a slightly better fit ( $\chi^{2}$ of 594.5 for 572 dof). We note that the line energy of this extra line gets close to the Ar XVII DR line at $3.62 \mathrm{keV}$. So we removed the extra Gaussian line and re-fit the Perseus spectrum removing the upper limits on the Ar XVII DR line. We obtained only a slightly worse fit than the previous case, with a $\chi^{2}$ of 598.8 (574 dof). The measured flux of the Ar XVII DR line at $3.62 \mathrm{keV}$ in this case was $4.8_{-0.8}^{+0.7}\left({ }_{-1.4}^{+1.3}\right) \times 10^{-5}$ photons $\mathrm{cm}^{-2} \mathrm{~s}^{-1}$, which is a factor of 30 above the predicted maximum flux of the Ar XVII DR line based on the measured flux of the Ar XVII line at $\sim 3.12 \mathrm{keV}$ and AtomDB line rates. The predicted maximum flux of the Ar XVII DR line for the Perseus spectrum was $1.6 \times 10^{-6}$ photons $\mathrm{cm}^{-2} \mathrm{~s}^{-1}$ $(<0.01$ times the flux of the Ar XVII triplet at $\sim 3.12$ $\mathrm{keV})$.

This test showed that the line detected in the Perseus Cluster could also be interpreted as an abnormally bright Ar XVII DR line. We note, however, that obtaining such a bright DR line relative to the He-like triplet at $3.12 \mathrm{keV}$ is problematic. The emissivity of the satellite line peaks at $\mathrm{kT}=1.8 \mathrm{keV}$, and declines sharply at lower temperatures, in addition to the change in the ionization balance which reduces the $\mathrm{Ar}^{+17}$ content of the plasma. The emissivity ratio for the $\mathrm{DR} / 3.12 \mathrm{keV}$ has its maximum value of 0.04 

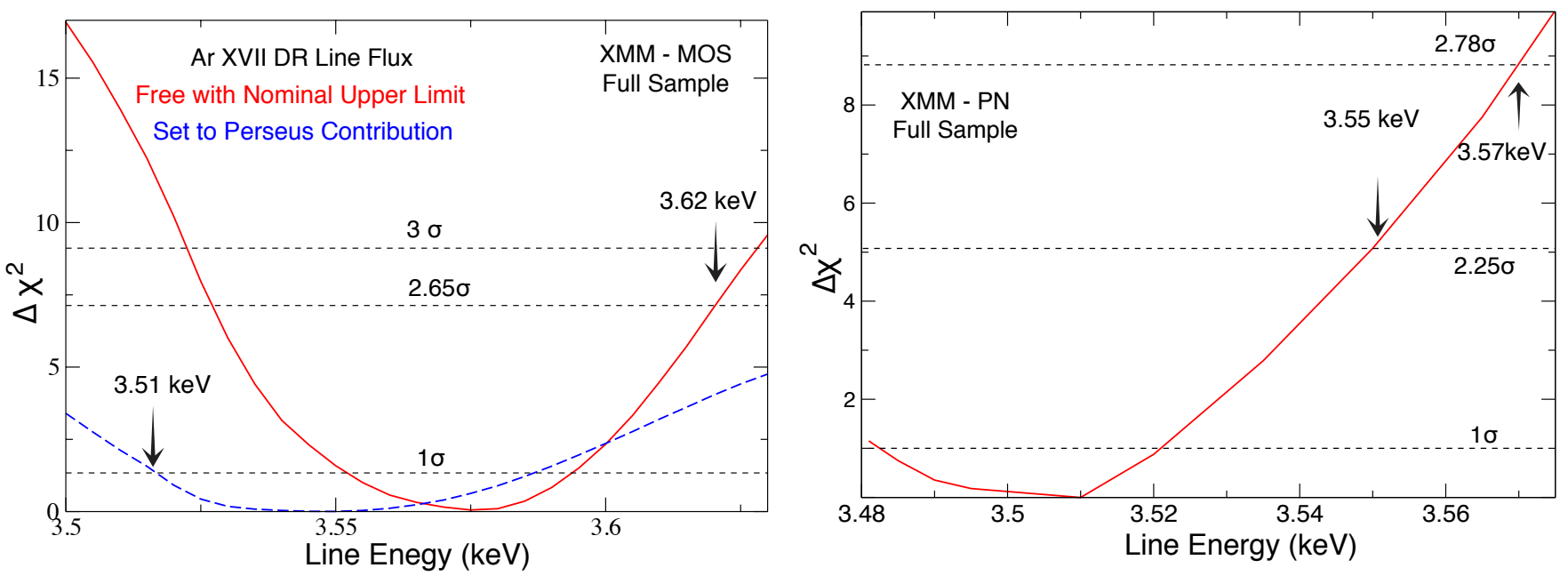

Figure 9. Left Panel: change in the goodness-of-fit statistics as a function of the detected line energy at 3.55-3.57 keV obtained from the stacked MOS observations of the full sample. The red solid line shows the confidence of the line energy when the flux of the Ar XVII DR line at $3.62 \mathrm{keV}$ was left free to vary within the AtomDB predicted boundaries. In this case, the detected line is $2.65 \sigma$ from the Ar XVII DR line at $3.62 \mathrm{keV}$ line. The blue dashed line shows the confidence curve of the line energy when the flux of the Ar XVII DR line at 3.62 $\mathrm{keV}$ was fixed at the maximum DR contribution from the Perseus Cluster. In this case the line energy is consistent with the PN detection. Right Panel: change in the goodness-of-fit statistics as a function of the line energy obtained from the stacked PN observations of the full sample. The line energy is $2.2 \sigma$ and $2.7 \sigma$ away from the MOS detections.

at $\mathrm{kT}=0.7 \mathrm{keV}$, but the emissivity of both lines is weak here, so any hotter component will dominate and lead to a lower ratio being observed.

To avoid cool gas in the Perseus core contaminating the flux of the nearby Ar and K lines, we also tried excising the central region with $1^{\prime}$ radius of the cluster and performed the fit on the core-excised co-added MOS spectrum. We found that adding an extra Gaussian line at $3.57 \mathrm{keV}$ has improved the fit by $\Delta \chi^{2}$ of 12.8 for an additional degree of freedom with a best-fit flux of $2.1_{-0.6}^{+0.7}$ $\left({ }_{-1.1}^{+1.2}\right) \times 10^{-5}$ photons $\mathrm{cm}^{-2} \mathrm{~s}^{-1}$ (see Figure 8). Excising the innermost $1^{\prime}$ reduced the flux of the detected line by a factor of two, indicating that the most of the flux of this emission originates from the cool core. The mixing angle that corresponds to the line flux from the core-excised Perseus spectrum is consistent within $1 \sigma-2 \sigma$ with those for the bright clusters (Centaurus+Coma+Ophiuchus) and the full sample, respectively (Table 5).

We also note that some scatter of the dark matter decay signal between individual clusters is naturally expected. For example, one can imagine a filament of dark matter along the line of sight in the direction of Perseus, which may boost the flux of the detected line and cause tension between the Perseus Cluster and the full sample. However, such a filament would have to be rather extreme.

\subsection{Refitting Full Sample with Anomalous $3.62 \mathrm{keV}$ Line}

With the knowledge that the $3.62 \mathrm{keV}$ line can be anomalously high (at least in Perseus), we should now try to re-fit the stacked MOS spectrum of the full sample to see if the line in the full sample is affected by the 3.62 $\mathrm{keV}$ excess from Perseus, which is part of the full sample. We set the flux of the $3.62 \mathrm{keV}$ line to the Perseus contribution of the Ar XVII DR line to the full-sample spectrum $\left(2.3 \times 10^{-6}\right.$ photons $\left.\mathrm{cm}^{-2} \mathrm{~s}^{-1}\right)$, assuming that all the new line flux in Perseus originates from the abnormally bright DR line. We note that this flux was already a factor of 30 above the predicted upper limits by AtomDB. Adding an extra Gaussian component, representing the new line, to a model with the anomalous $3.62 \mathrm{keV}$ line still improves the fit by $\Delta \chi^{2}$ of 6.52 for 2 dof. The bestfit energy and flux were $3.55 \pm 0.03(0.05)$ and $2.2_{-0.9}^{+1.6}$ $\left({ }_{-1.5}^{+2.2}\right) \times 10^{-5}$ photons $\mathrm{cm}^{-2} \mathrm{~s}^{-1}$, respectively. The new line is still required with $2.5 \sigma$ in the full sample; however, the energy of this line gets lower and its confidence interval wider. The line energy comes into agreement with the energy detected in the PN full sample (see Figure 9. left panel). If we completely free the normalization of the $3.62 \mathrm{keV}$ line in the full-sample MOS spectrum, it becomes lower than the Perseus contribution that we considered above.

\section{CHANDRA OBSERVATIONS OF PERSEUS AND VIRGO}

Due to the potential significance of the discovery of an emission line due to the decay of sterile neutrinos in clusters, it is necessary to confirm it with another instrument. Pending a full stacking analysis of the Chandra and Suzaku cluster archives (which is a current work in progress), we analyze two Chandra observations of the Perseus Cluster and Virgo Cluster, which have over 1 Ms and $500 \mathrm{ks}$ of total Chandra exposure, respectively. A summary of the Chandra observations used in this work to confirm the detection is given in Table 6 .

The Chandra ACIS-I and ACIS-S data were processed following Vikhlinin et al. (2005), using CIAO 4.5 and CALDB 4.5.7. Each event list was filtered for high background periods. After this filtering the total good times were $487 \mathrm{ks}$ and $883 \mathrm{ks}$ for the ACIS-I and ACIS-S observations of the Perseus Cluster core, respectively. We have extracted the ACIS-S spectra from the full S3 chip excluding the $1^{\prime}$ region surrounding the cluster centroid and one of the observations (ID: 4950) with a background flare. The ACIS-I spectrum was extracted using a circular region covering the full ACIS-I FOV. The filtered 
Table 6

Summary of Chandra Observations of the Perseus Cluster and Virgo Cluster used in This Work. The columns list (1) primary detector array used; (2) observation number (3) and (4) right ascension and declination of the pointing (J2000); (5) good exposure time in ks after filtering.

\begin{tabular}{|c|c|c|c|c|c|c|}
\hline $\begin{array}{l}\text { (1) } \\
\text { Cluster }\end{array}$ & $\begin{array}{c}(2) \\
\text { Detector }\end{array}$ & $\begin{array}{c}(3) \\
\text { Obs ID }\end{array}$ & $\begin{array}{l}(4) \\
\text { RA }\end{array}$ & $\begin{array}{c}(5) \\
\mathrm{DEC}\end{array}$ & Exposure (ks) & Redshift \\
\hline \multirow[t]{8}{*}{ Perseus } & ACIS-I & 11713 & 031931.8 & +413749.0 & 113.0 & 0.017 \\
\hline & ACIS-I & 11714 & 031942.6 & +413407.0 & 92.3 & 0.017 \\
\hline & ACIS-I & 11715 & 031944.2 & +412518.0 & 73.6 & 0.019 \\
\hline & ACIS-I & 11716 & 031944.2 & +412518.0 & 39.4 & 0.017 \\
\hline & ACIS-I & 12025 & 031931.8 & +413749.0 & 17.6 & 0.017 \\
\hline & ACIS-I & 12033 & 031931.8 & +413749.0 & 18.6 & 0.018 \\
\hline & ACIS-I & 12036 & 031931.8 & +413749.0 & 47.7 & 0.018 \\
\hline & ACIS-I & 12037 & 031944.2 & +412518.0 & 85.0 & 0.018 \\
\hline \multirow[t]{12}{*}{ Perseus } & ACIS-S & 4289 & 031947.6 & +413037.0 & 95.4 & 0.018 \\
\hline & ACIS-S & 3209 & 031947.6 & +413037.0 & 95.7 & 0.018 \\
\hline & ACIS-S & 4946 & 031948.2 & +413042.2 & 23.6 & 0.018 \\
\hline & ACIS-S & 6139 & 031948.2 & +413042.2 & 56.4 & 0.018 \\
\hline & ACIS-S & 4947 & 031948.2 & +413042.2 & 29.7 & 0.018 \\
\hline & ACIS-S & 6145 & 031948.2 & +413042.2 & 85.0 & 0.018 \\
\hline & ACIS-S & 4948 & 031948.2 & +413042.2 & 118.6 & 0.018 \\
\hline & ACIS-S & 4949 & 031948.2 & +413042.2 & 29.4 & 0.018 \\
\hline & ACIS-S & 6146 & 031948.2 & +413042.2 & 47.1 & 0.018 \\
\hline & ACIS-S & 4951 & 031948.2 & +413042.2 & 96.1 & 0.018 \\
\hline & ACIS-S & 4952 & 031948.2 & +413042.2 & 164.2 & 0.018 \\
\hline & ACIS-S & 4953 & 031948.2 & +413042.2 & 30.1 & 0.018 \\
\hline \multirow[t]{7}{*}{ Virgo } & ACIS-I & 5826 & 123049.5 & +122328.0 & 127.5 & 0.0040 \\
\hline & ACIS-I & 5827 & 123049.5 & +122328.0 & 157.6 & 0.0038 \\
\hline & ACIS-I & 5828 & 123049.5 & +122328.0 & 33.2 & 0.0036 \\
\hline & ACIS-I & 6186 & 123049.5 & +122328.0 & 50.8 & 0.0040 \\
\hline & ACIS-I & 7210 & 123049.5 & +122328.0 & 31.1 & 0.0033 \\
\hline & ACIS-I & 7211 & 123049.5 & +122328.0 & 15.5 & 0.0038 \\
\hline & ACIS-I & 7212 & 123049.5 & +122328.0 & 65.3 & 0.0036 \\
\hline
\end{tabular}

ACIS-I good time for the Virgo Cluster core was $481 \mathrm{ks}$. The radius of $500^{\prime \prime}$ was used for extraction of ACIS-I spectra of the Virgo Cluster. Analysis steps include image creation, point-source detection with wavdetect, and their removal.

Background corrections were made using the blank-sky background fields, including the "period-E" background files. For each target event file, a corresponding background event file was generated and normalized by the ratio of counts in the $9.0-12.0 \mathrm{keV}$ energy range (Hickox \& Markevitch 2006). Because we are interested in the high-energy part of the spectrum, modeling of the soft sky CXB is not relevant.

\subsection{Chandra Data Analysis}

Each spectrum was fitted using a standard multitemperature apec model as described in Section 2.3 to determine the best-fit X-ray redshift of each observation, shown in Table 6. Each event file was then blue-shifted to the cluster's source frame using these best-fit redshifts. The source and background spectra in the source's frame were obtained by generating spectra using the scaled event energy values in the event files. The ARFs and RMFs were remapped based on the estimates of the best- fit redshifts. The RMFs and ARFs were weighted by only the exposure time of each observation. The scaled source and background spectra were co-added using the FTOOL mathpha, whereas ARFs and RMFs were merged using the FTOOLS addarf and addrmf tools, respectively.

\subsection{Chandra Detection of the Emission Line in Perseus}

Following the same method as described in Section 3.1. the continuum emission was fit using the line-free apec model with additional Gaussian models to represent the strong emission lines. The best-fit temperature from the $2.0-6.0 \mathrm{keV}$ band and normalizations of the line-free apec model, fluxes, and equivalent widths of $\mathrm{S} x \mathrm{XVI}, \mathrm{Ca}$ XIX, and Ca Xx lines at $2.63 \mathrm{keV}, 3.90 \mathrm{keV}$, and $4.11 \mathrm{keV}$ are given in Table 7. We have searched especially the 3.0 - $4.0 \mathrm{keV}$ interval where the $3.57 \mathrm{keV}$ line emission was detected in the stacked XMM-Newton observations. The measured fluxes of S XVI, Ca XIX, and Ca XX lines from the ACIS-I and ACIS-S spectra with the AtomDB fluxes yielded the maximum predicted fluxes of $\mathrm{K}$ XVIII lines at $3.47 \mathrm{keV}$ and $3.51 \mathrm{keV}$, Ar XVII line at $3.68 \mathrm{keV}$, and $\mathrm{K}$ XVIII line at $3.71 \mathrm{keV}$ as described in detail in Section 3.1. The triplet emission line at Ar XVII $3.12 \mathrm{keV}$ was used to determine the maximum allowed flux of the $\mathrm{Ar}$ 

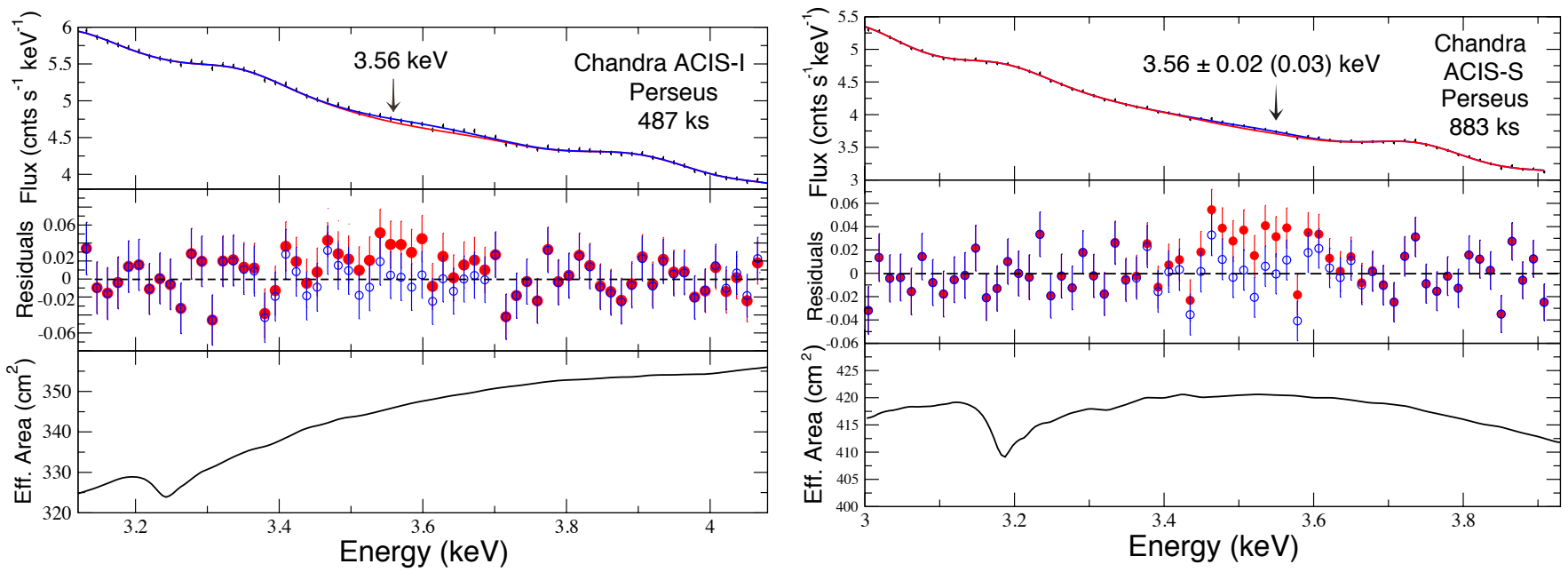

Figure 10. Close-up $3.1-4.1 \mathrm{keV}$ energy interval of the co-added spectrum obtained from Chandra ACIS-I and ACIS-S observations of the Perseus Cluster. The continuum emission was fit with the $2 \mathrm{~T}$ line-free apec model, while emission lines were modeled with additional Gaussian components. The K XVIII (3.51 keV), Ar XVII $(3.62 \mathrm{keV})$, and Ar XVII $(3.68 \mathrm{keV})$ lines are also included in the total model shown as a red line on the top panel. The blue line shows the total model after a Gaussian component is added to the total model, indicating that the weak residual can be modeled with a Gaussian. The bottom panels show the weak residual before and after the Gaussian is added to the total model.

Table 7

Best-fit Temperature and Normalizations of line-free apec Model Fit to the Co-added Chandra Observations of the Perseus and Virgo Clusters. Fluxes of the S XVI, Ca XIX, Ca Xx at the rest energies $2.63 \mathrm{keV}, 3.90 \mathrm{keV}, 4.11 \mathrm{keV}$ are given.

\begin{tabular}{|c|c|c|c|}
\hline Model Paramaters & $\begin{array}{l}\text { Perseus } \\
\text { ACIS-I }\end{array}$ & ACIS-S & $\begin{array}{c}\text { Virgo } \\
\text { ACIS-I }\end{array}$ \\
\hline $\mathrm{kT}_{1}(\mathrm{keV})$ & $4.58 \pm 0.07$ & $2.77 \pm 0.18$ & $1.18 \pm 0.07$ \\
\hline$N_{1}\left(10^{-1} \mathrm{~cm}^{-5}\right)$ & $1.20 \pm 0.01$ & $3.21 \pm 0.35$ & $2.22 \pm 0.10$ \\
\hline $\mathrm{kT}_{2}(\mathrm{keV})$ & $5.34 \pm 0.02$ & $4.79 \pm 0.18$ & $5.08 \pm 0.37$ \\
\hline$N_{2}\left(10^{-1} \mathrm{~cm}^{-5}\right)$ & $3.83 \pm 0.13$ & $2.31 \pm 0.24$ & $1.02 \pm 0.23$ \\
\hline Flux of S XVI $\left(10^{-4} \mathrm{pht} \mathrm{cm}^{-2} \mathrm{~s}^{-1}\right)$ & $3.94 \pm 0.15$ & $3.72 \pm 0.85$ & $6.18 \pm 0.13$ \\
\hline 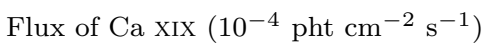 & $1.29 \pm 0.08$ & $1.05 \pm 0.08$ & $1.22 \pm 0.88$ \\
\hline Flux of Ca xx $\left(10^{-4}\right.$ pht $\left.\mathrm{cm}^{-2} \mathrm{~s}^{-1}\right)$ & $1.10 \pm 0.05$ & $1.07 \pm 0.05$ & $0.35 \pm 0.05$ \\
\hline
\end{tabular}

XVII DR line at $3.62 \mathrm{keV}$ at any plasma temperature as described above. The predicted fluxes of these lines are given in Table 8 . Using 0.1 and 3 times of the upper bound of these estimates as lower and upper limits for $\mathrm{K}$ XVIII andAr XVII, and $10^{-3}-10^{-2}$ times the flux of the Ar XVII triplet for the lower and upper bounds for the Ar XVII DR line, we determined the best-fit flux of the weak residual around $3.57 \mathrm{keV}$.

An additional Gaussian model improves the fit by $\Delta \chi^{2}$ of 11.8 for an additional 2 dof. The line was unresolved and consistent with broadening by the instrument response in the Perseus Cluster spectra. The Perseus ACIS-S spectra yield a best-fit energy of $3.56 \pm 0.02$ (0.03) keV for an additional Gaussian model, given in Table 5 . The flux of the detected signal is $1.02_{-0.4}^{+0.4}\left({ }_{-0.5}^{+0.5}\right)$ $\times 10^{-5}$ photons $\mathrm{cm}^{-2} \mathrm{~s}^{-1}$. This detection corresponds to a false detection probability of $0.5 \%$ in the co-added ACIS-S spectrum. The right panel of the Figure 10 shows the signal in the Chandra ACIS-S observations of the Perseus Cluster before and after the Gaussian model is added to the fit.

To further demonstrate that the detected flux is independent of the spectral modeling, we fit the ACIS-S spectrum of the Perseus Cluster with a two-temperature vapec model with abundances of trace elements set to that of Fe. We obtained an acceptable fit in the $3-$ $6 \mathrm{keV}$ energy band with $\chi^{2}$ of 182.1 for 147 dof. An additional Gaussian model at $3.56 \mathrm{keV}$ (rest energy) improved the fit by $\Delta \chi^{2}$ of 16 for an extra degree of freedom. The best-fit flux of the line is $1.09 \pm 0.26(0.42)$ $\times 10^{-5}$ photons $\mathrm{cm}^{2} \mathrm{~s}^{-1}$, which is consistent with the flux measured in the line-free apec model fit with additional Gaussian models. This test shows that the detection is robust and independent of the method used in the spectrum modeling. The Perseus co-added spectrum fit with a two-temperature vapec model is shown in Figure 11.

We then performed the same search in the co-added 
Table 8

Best-fit Temperature and Normalizations of line-free apec Model Fit to the Co-added Chandra Spectra of Perseus and Virgo Clusters. (1) and (2) are the estimated fluxes of K XVIII at the rest energy $3.51 \mathrm{keV}$ and Ar XVII at the rest energy $3.68 \mathrm{keV}$ lines obtained from AtomDB in the units of photons $\mathrm{cm}^{-2} \mathrm{~s}^{-1} ;(3)$ and (4) are the estimated energy in $\mathrm{keV}$ and flux of the unidentified emission line in the units of photons $\mathrm{cm}^{-2} \mathrm{~s}^{-1} ;(5)$ is the measured equivalent width of the spectral feature, indicates the ratio of line flux to continuum flux in the units of $\mathrm{keV}$.

\begin{tabular}{|c|c|c|c|c|c|c|}
\hline Cluster & Inst. & $\begin{array}{c}\text { Flux } \\
\text { K XVIII } \\
(3.47 \mathrm{keV}) \\
\left(10^{-6}\right)\end{array}$ & $\begin{array}{c}\text { Flux } \\
\text { K XVIII } \\
(3.51 \mathrm{keV}) \\
\left(10^{-6}\right)\end{array}$ & $\begin{array}{c}\text { Flux } \\
\text { Ar XvII } \\
(3.62 \mathrm{keV}) \\
\left(10^{-7}\right)\end{array}$ & $\begin{array}{c}\text { Flux } \\
\text { Ar XVII } \\
(3.68 \mathrm{keV}) \\
\left(10^{-5}\right)\end{array}$ & $\begin{array}{c}\text { Flux } \\
\text { K xix } \\
(3.71 \mathrm{keV}) \\
\left(10^{-6}\right)\end{array}$ \\
\hline \multirow{3}{*}{ Perseus } & ACIS-I & $3.4 \pm 2.7$ & $3.1 \pm 2.4$ & $4.3 \pm 3.5$ & $0.8 \pm 0.4$ & $2.6 \pm 2.1$ \\
\hline & & & & & & \\
\hline & ACIS-S & $4.5 \pm 2.3$ & $4.15 \pm 2.2$ & $5.8 \pm 2.8$ & $1.3 \pm 1.0$ & $3.4 \pm 2.7$ \\
\hline Virgo & ACIS-I & $2.0 \pm 0.5$ & $3.6 \pm 1.0$ & $38.2 \pm 10.6$ & $1.7 \pm 0.5$ & $1.8 \pm 0.6$ \\
\hline
\end{tabular}

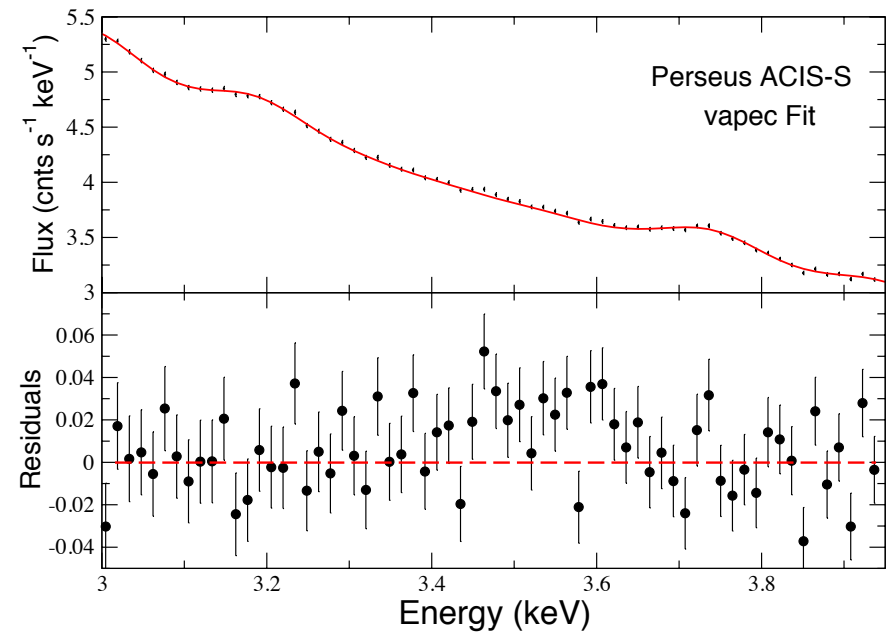

Figure 11. The $3-4 \mathrm{keV}$ energy interval for the co-added spectrum obtained from Chandra ACIS-S observations of the Perseus Cluster. The continuum emission was fit with two vapec models. This detection demonstrates that the detected line is not a fitting artifact.

ACIS-I spectrum of the Perseus Cluster. Fitting the 2.5$6 \mathrm{keV}$ band of the ACIS-I spectrum with a line-free apec model with additional Gaussian lines as described above produced a good fit overall with a total $\chi^{2}$ of 158.7 for 152 dof. Adding a Gaussian line at $3.56 \mathrm{keV}$, the energy where the line was detected in the co-added ACIS$\mathrm{S}$ spectrum of the Perseus Cluster, improved the fit by $\Delta \chi^{2}$ of 6.2 for an additional degree of freedom. The flux of the detected signal was $1.9_{-0.8}^{+0.8}\left({ }_{-1.6}^{+1.2}\right) \times 10^{-5}$ photons $\mathrm{cm}^{-2} \mathrm{~s}^{-1}$ in the co-added ACIS-I spectrum. The left panel of the Figure 10 shows the ACIS-I spectrum of the Perseus Cluster before and after an additional Gaussian model is added to the total model, to demonstrate the detection of the line.

The mixing angle $\sin ^{2}(2 \theta)$ estimate from the co-added Chandra ACIS-S observations of the Perseus Cluster is $4.0_{-1.4}^{+1.5}\left({ }_{-1.8}^{+1.8}\right) \times 10^{-10}$, which is consistent with the angle obtained from the co-added ACIS-I and XMM-Newton MOS observations of the Perseus Cluster at the $1 \sigma$ level.

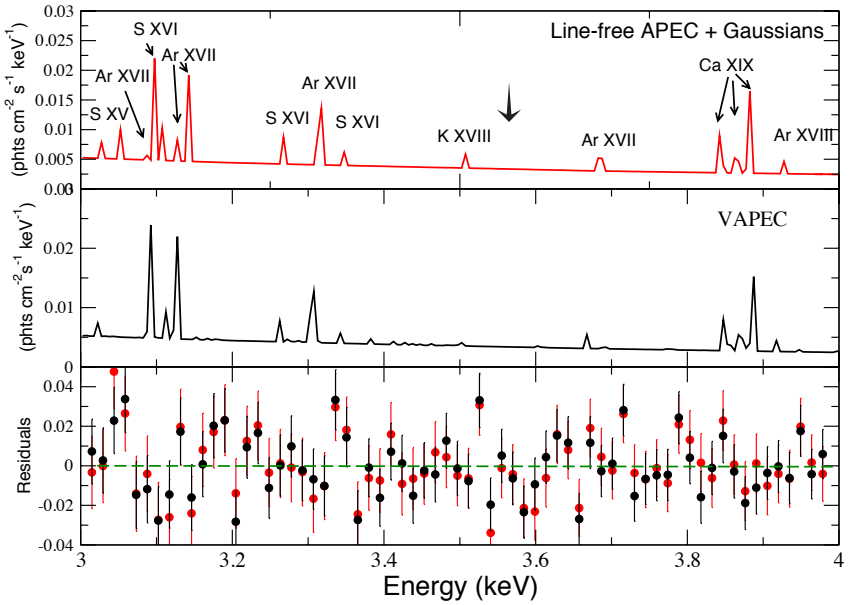

Figure 12. $3-4 \mathrm{keV}$ energy interval for the co-added spectrum obtained from Chandra ACIS-I observations of the Virgo Cluster at the redshifted frame. The continuum emission was fit with the line-free apec model with Gaussians components (upper panel) and two-temperature vapec models (middle panel). The lower panel shows the differences in the residuals for the two models. Red data points show the residuals of the line-free apec model with Gaussians components and black data points show the residuals of the vapec model. The energy where the line is detected in the Chandra ACIS observations of the Perseus Cluster is indicated with an arrow.

Since the ACIS-S chip covers the central 4'region of the Perseus core, higher flux measured from ACIS-S observations also indicates that this emission is concentrated in the core, confirming the results from the XMM-Newton observations of the Perseus core.

\subsection{Chandra Upper Limit on the Line from Virgo}

We have performed the same fitting strategy described above to the co-added spectra of the Virgo Cluster, e.g., line-free apec model with additional Gaussian lines. We used the lower and upper limits to the $\mathrm{K}$ and Ar line in the $3.4-3.7 \mathrm{keV}$ band based on the upper limits estimated from AtomDB (given in Table 8). The overall fit was acceptable with a total $\chi^{2}$ of 82.5 for 62 dof. Unlike the Perseus Cluster, the co-added Virgo Cluster did not show any residuals around $3.57 \mathrm{keV}$ in the fit 
with the line-free apec model. Adding a Gaussian line did not significantly improve the fit. We were able to place an upper limit of $9.1 \times 10^{-6}$ photons $\mathrm{cm}^{-2} \mathrm{~s}^{-1}$ at the $90 \%$ confidence level. This limit corresponds to an upper limit on the mixing angle of $\sin ^{2}(2 \theta)<1.1 \times$ $10^{-10}$.

We also fit the $2.5-4.0 \mathrm{keV}$ band of the Virgo spectrum using a two-temperature standard vapec model. The fit has a total $\chi^{2}$ obtained from the vapec model of 91.7 for 82 dof. We overall obtained a better fit with the standard vapec model than the fit with the line-free apec model. The best-fit model also did not require the addition of a line at $3.56 \mathrm{keV}$. The $90 \%$ upper limit to the flux of this line is $<6.2 \times 10^{-6}$ photons $\mathrm{cm}^{-2} \mathrm{~s}^{-1}$. The differences in the modeling approaches used in the ACIS-I spectrum fits of the Virgo Cluster (line-free apec with Gaussians and vapec) are demonstrated in Figure 12 . The factor of two difference in the upper limits on the flux measurements indicates that the systematical uncertainties in the flux measurements can be as large as a factor of two depending on the modeling method used in this analysis.

\section{DISCUSSION}

Stacking X-ray spectra of galaxy clusters from different redshifts can make it possible to detect weak emission features. This method was successfully used before; for example, Sanders \& Fabian (2011) the stacked XMMNewton Reflection Grating Spectrometer (RGS) observations of 62 clusters to find evidence of OVII in cluster cores. (The RGS energy coverage is limited to $E<2$ $\mathrm{keV}$.) In this work, we stacked the XMM-Newton MOS (6 $\mathrm{Ms})$ and PN (2 Ms) spectra of 73 nearby $(z<0.35)$ wellexposed galaxy clusters to look for any unknown emission lines at $E>2 \mathrm{keV}$. The redshift difference in our full cluster sample corresponds to an energy difference of up to $1.2 \mathrm{keV}$ at $E \sim 3.5 \mathrm{keV}$ and still more at higher energies. Thus, when the cluster spectra are stacked in the source reference frame, any uncalibrated detector response features or instrumental and background lines are smeared by this difference in energy, minimizing their effects and making it possible to detect the lines in the source spectrum that for any single object would be below the level of detector artifacts.

We have detected a weak line at the rest energy of $3.57 \pm 0.02 \mathrm{keV}$ (68\% statistical confidence interval) in XMM-Newton MOS observations. A similar emission line has been found independently in the stacked PN spectrum of the full sample, although its best-fit energy was lower, $3.51 \pm 0.03 \mathrm{keV}$. There is tension between these energies at a $2.8 \sigma$ level, including only statistical errors; they become consistent once we introduce another degree of freedom in the model.

The best-fit fluxes of $4.0_{-0.8}^{+0.8} \times 10^{-6}$ photons $\mathrm{cm}^{-2} \mathrm{~s}^{-1}$ and $3.9_{-1.0}^{+0.6} \times 10^{-6}$ photons $\mathrm{cm}^{-2} \mathrm{~s}^{-1}$ obtained from the stacked MOS and PN observations of the full sample are consistent with each other. This emission line has been found in a blind search in $\sim 70$ independent energy bins. Even taking into account the "look-elsewhere" effect (the probability of finding a line in any of those bins), the statistical probability of a false detection of such a $4-5 \sigma$ line at the same energy in two statistically independent data sets is negligibly small.

To see if the signal originates from one of the dominant nearby clusters in the sample, we divided the full sample into three subsamples - Perseus, Centaurus + Coma + Ophiuchus, and all others - and detected the line in all three. In the Centaurus + Coma + Ophiuchus MOS spectrum, the line was found with a flux of $1.6_{-0.4}^{+0.3} \times 10^{-5}$ photons $\mathrm{cm}^{-2} \mathrm{~s}^{-1}$ at $3.57 \mathrm{keV}$. The lower signal-to-noise ratio (128 ks total) PN spectrum yielded a non-detection, with a $90 \%$ upper limit of $9.5 \times 10^{-6}$ photons $\mathrm{cm}^{-2} \mathrm{~s}^{-1}$.

The stacked MOS and PN observations in the rest frame of the fainter 69 "all other" clusters exhibited the emission line at $3.57 \mathrm{keV}$ line with the best-fit flux of $2.1_{-0.5}^{+0.4} \times 10^{-6}$ and $2.0_{-0.5}^{+0.3} \times 10^{-6}$ photons $\mathrm{cm}^{-2} \mathrm{~s}^{-1}$ at the energy $3.57 \mathrm{keV}$. Thus, the significant detection of the line does not originate from one or a few dominant clusters, but is produced by all the clusters in subsamples.

We investigated the spectrum of the Perseus Cluster in greatest detail, because it contributes a large fraction of the total number of photons, and because the line in Perseus significantly deviates from scaling by cluster mass (implied by one of our explanations for this line). The full-FOV MOS spectrum of Perseus shows a line with the best-fit energy of $3.57 \mathrm{keV}$ line and a flux of $5.2_{-1.5}^{+2.4} \times 10^{-5}$ photons $\mathrm{cm}^{-2} \mathrm{~s}^{-1}$; the addition of a line improves the fit by $\Delta \chi^{2}=15.7$ for an additional degree of freedom. We note that the flux of the detected line is dependent on the predicted fluxes of the nearby K XVIII triplet at $3.51 \mathrm{keV}$ and an Ar XVII DR line at $3.62 \mathrm{keV}$. In the spectral fits of the Perseus Cluster, the fluxes of these nearby lines were at their allowed upper limits. Relaxing these upper limits shifts the best fit line energy to 3.59 $\mathrm{keV}$, suggesting that the detected line could be the Ar XVII DR line at $3.62 \mathrm{keV}$. To test this, we removed the line model component at $3.57 \mathrm{keV}$, instead letting the $3.62 \mathrm{keV}$ line have arbitrary normalization, and found that the Perseus spectra could be represented without an additional line. However, in this case, the implied flux of the Ar XVII DR line had to be significantly increased from the AtomDB estimate, which is $1 \%$ of the strength of the Ar XVII triplet, to $30 \%$. Physically, it is difficult to create such a bright Ar XVII DR line relative to the Ar XVII He-like triplet at $3.12 \mathrm{keV}$. The emissivity ratio for the Ar XVII DR line to the Ar XVII triplet at 3.12 $\mathrm{keV}$ has its maximum value of $4 \%$ at $\mathrm{kT}=0.7 \mathrm{keV}$. Since the emissivity of both lines is weak at this temperature, any hotter temperature components (which produce the bulk of the cluster emission) will dominate the spectra, leading to an even lower observed normalization ratio. To explain our line, the required flux of the Ar XVII DR line has to be increased from the AtomDB estimate by an implausible factor $\sim 30$. This possibility is further discussed in Section 5.1 .

To further investigate the origin of this excess emission, we excluded the central bright and complex region with $1^{\prime}$ radius of the Perseus core. The best-fit flux of $2.1_{-0.6}^{+0.7}$ $\times 10^{-5}$ photons $\mathrm{cm}^{-2} \mathrm{~s}^{-1}$ at $3.57 \mathrm{keV}$ decreased to half of the full cluster flux. This decrease indicates that the emission is concentrated in the immediate cool core of the Perseus Cluster.

In addition, we investigated the Chandra ACIS-S and ACIS-I spectra of Perseus to confirm that the detected signal is not an XMM-Newton detector feature. An inde- 
pendent search of the $3.0-4.0 \mathrm{keV}$ interval of the ACIS spectra revealed a positive detection of the feature at a significance of $3.4 \sigma$ and $2.5 \sigma$ for the line energy given by XMM-Newton fits. The measured best-fit energy of 3.56 $\pm 0.02 \mathrm{keV}$ in the ACIS-S spectrum (with the $1^{\prime}$ central region of the core excised) is consistent with the best-fit energy obtained from the stacked XMM-Newton observations of the full sample. The observed flux of the detected feature is $1.0_{-0.4}^{+0.4} \times 10^{-5}$ photons $\mathrm{cm}^{-2} \mathrm{~s}^{-1}$. The same feature was also observed in the co-added ACIS-I spectrum of the Perseus Cluster with the best-fit flux of $1.8_{-0.8}^{+0.8} \times 10^{-5}$ photons $\mathrm{cm}^{-2} \mathrm{~s}^{-1}$, with a lower significance $\left(\Delta \chi^{2}=6.2\right.$ for 1 dof $)$. However, the feature was not detected in the Chandra ACIS-I observations of the Virgo Cluster, for which we placed a $90 \%$ upper limit of $9.1 \times 10^{-6}$ photons $\mathrm{cm}^{-2} \mathrm{~s}^{-1}$.

\subsection{Unknown Plasma Emission Line?}

One possible interpretation is that the detected line is an unknown plasma emission line. The flux of the line corresponds to a maximum emissivity of $3.3 \times 10^{-18}$ photons $\mathrm{cm}^{3} \mathrm{~s}^{-1}$, derived using the emission measure appropriate for the lowest temperature $(4.36 \mathrm{keV}) \mathrm{com}-$ ponent as described in Section 3.1. For comparison, this is similar to the maximum emissivity of the $\mathrm{Ca} \mathrm{xx} \mathrm{Ly} \alpha$ line at $4.1 \mathrm{keV}$. Given that the $\mathrm{Ca}$ xx line was previously observed in individual galaxy cluster spectra, including the Perseus Cluster (e.g. Tamura et al. 2009), a line as strong at $\sim 3.57 \mathrm{keV}$ would have been observed had it been expected. However, there is no likely candidate for an atomic transition near $3.57 \mathrm{keV}$. The emission lines of strong hydrogen- and helium-like ions are well known, and none fall in this band. The only candidate emission line from such an ion would be the He-like Cl XVI $n=5 \rightarrow 1$ transition at $3.52 \mathrm{keV}$, but if this is the cause it would imply the presence of even stronger lines from the $n=3 \rightarrow 1$ and $n=4 \rightarrow 1$ transitions at 3.27 and $3.44 \mathrm{keV}$, respectively, but these are not seen. Emission lines from $L$-shell ions form a far more complex pattern. However, the binding energy of Li-like $\mathrm{Zn}(Z=30)$ is only $2.782 \mathrm{keV}$, so the transition lines of all lighter elements or less ionized species must be at lower energies than this. If this line is a K-shell fluorescence transition, it must be from an element whose neutral and Li-like $\mathrm{K}$ shell fluorescent line energies bound $3.57 \mathrm{keV}$. The only such atoms are argon and potassium, but in this case the relevant Ar K-shell fluorescence transition is simply another name for the Ar XVII DR line discussed in detail above. The neutral potassium $\mathrm{K} \alpha$ fluorescence line is at $3.313 \mathrm{keV}$, while neutral $\mathrm{K} \beta$ is at $3.59 \mathrm{keV}$, so there must be transitions at the relevant energy. In this case, the best matches are the K $\alpha$ transitions of K XVI through $\mathrm{K}$ XIV ions, which occur at $\sim 3.57 \mathrm{keV}$ (Palmeri et al. 2012). However, since at any temperature above $1 \mathrm{keV}$ potassium will have at most 2 bound electrons, any such line would have to be originating from an unknown source of photoionized potassium in clusters. Thus this scenario is very unlikely, since the compact sources (e.g., active galactic nuclei) are not strong enough to photoionize the low-density ICM.

Although a complete analysis was not shown, adding an Ar XVII DR line at $3.62 \mathrm{keV}$ with unconstrained flux into all of our spectra would significantly impact both the fit results and detection level of a line at 3.57 $\mathrm{keV}$. We have constrained this line to be at most $1 \%$ of the strength of the unresolved Ar XVII triplet at 3.12 $\mathrm{keV}$, but must consider the physical situation required to maximize the $3.62 \mathrm{keV}$ DR line. In thermal equilibrium, the maximum strength of this line is $4 \%$ of the Ar XVII triplet, albeit at a temperature where the expected emission is negligible. One might also consider an extreme non-equilibrium situation with cold electrons that are unable to collisionally excite any Ar XVII lines, but dielectronic recombination is still possible. Examining the satellite line data in AtomDB, taken from Vainshtein \& Safronova (1980), shows that even in this case the maximum ratio is only $7 \%$, as there are DR satellite lines at the energies of the Ar XVII triplet as well and these lines would also be excited in such a case. While not physically impossible if there was a significant and unexpected error in the atomic physics calculations, we have no reason to believe this has occurred.

One other possibility is a radiative recombination continuum (RRC) edge feature. The S XVI recombination edge lies at $3.494 \mathrm{keV}$, and if it was bright enough, it might fill in some of the flux in this region. However, we note that the use of the no-line model has already included the RRC feature under equilibrium conditions. Producing a stronger RRC would require a sharp drop in the electron temperature, while retaining a large, fully stripped $\mathrm{S}^{16+}$ population. Also, if the temperature drops below $\approx 0.1 \mathrm{keV}$, the RRC feature becomes very narrow and will be an order of magnitude less powerful at 3.57 $\mathrm{keV}$ compared with right at the edge: this shape is not consistent with our observations. Similarly, at hotter temperatures, the RRC becomes almost constant with energy once above the edge. If the $\mathrm{RRC}$ was really there, we would expect to see a residual at about $3.6 \mathrm{keV}$, which we do not. Finally, we note that the edge is $50-80 \mathrm{eV}$ from our proposed line, which makes an unlikely source of the line.

We also note that our assumptions regarding relative line strengths have assumed that the ICM is in thermal equilibrium or close to it. Charge exchange (CX) between highly ionized ions and neutral hydrogen or helium could also create X-ray emission lines with different ratios (Smith et al. 2012). This could affect our assumption of equilibrium line ratios, although we have included a substantial range around the equilibrium values. It is important to note that these CX lines are not new, but rather the same lines occurring in different ratios. Due to its large cross section relative to electron excitation rates, astrophysical CX can occur only in a thin sheet where ions and neutrals interact directly, limiting its total emission relative to the large ICM volume. In certain cases, such as the core of the Perseus Cluster where many neutral filaments are known, it is possible that CX could be large enough to create a small fraction of the total Xray emission, although it would not create or enhance a line at $3.57 \mathrm{keV}$ or the DR line at $3.62 \mathrm{keV}$. CX could not dominate the overall emission, however, as it would also create Fe XVII and other lines that are not detected.

\subsection{Sterile neutrino decay line?}

An interesting interpretation of the line is the decay signature of the sterile neutrino, a long-sought dark matter particle candidate (Boyarsky et al. (e.g., 2009), see 

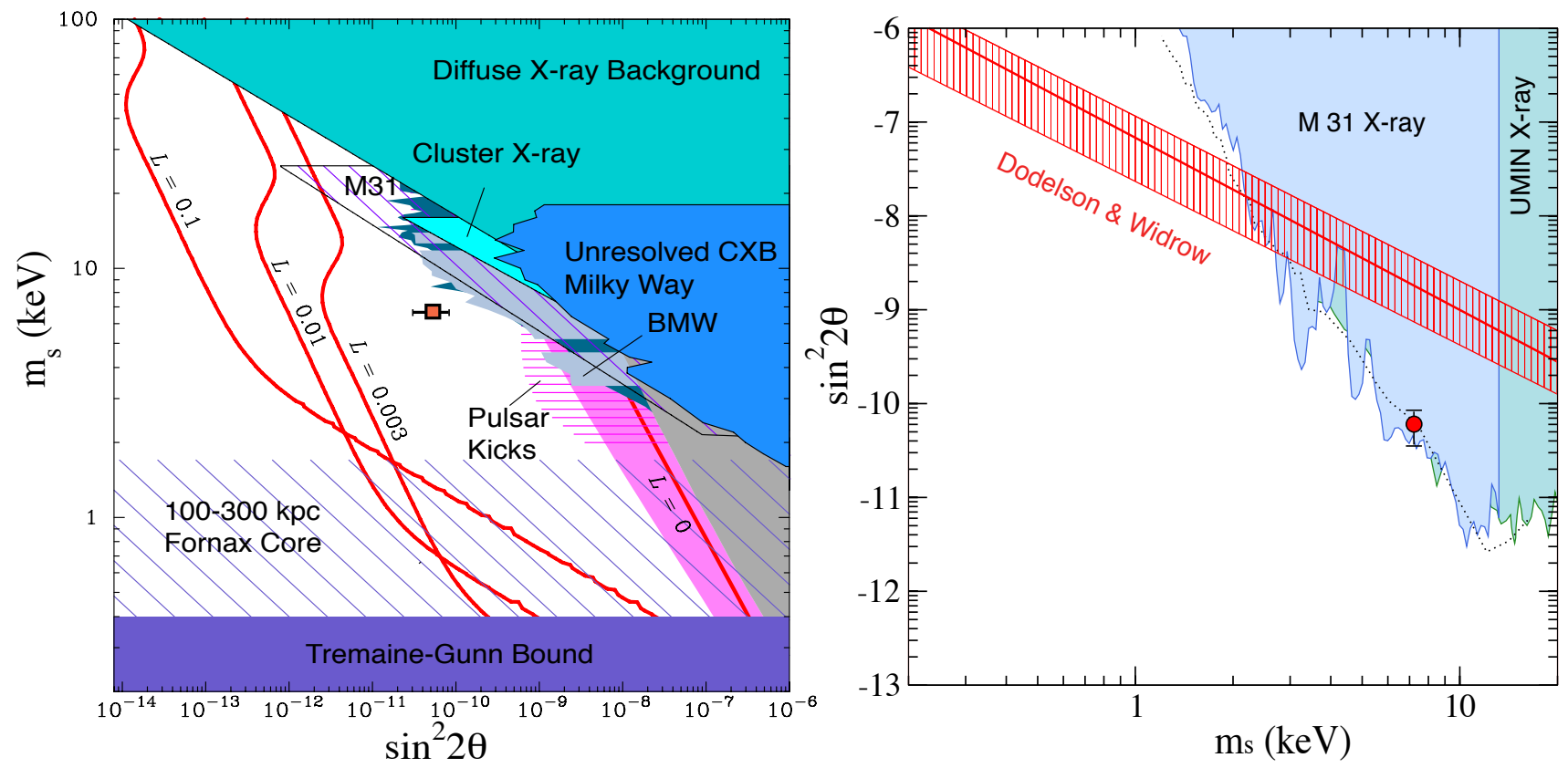

Figure 13. Constraints on the sterile neutrino model from the literature. Our full-sample MOS line detection (assuming that the line is from sterile neutrino and that all dark matter is in sterile neutrino) is shown by red symbols in both panels; error bar is statistical 90\%. Left: historic constraints from Abazajian (2009). Red curves show theoretical predictions for the Dodelson-Widrow mechanism assuming sterile neutrinos constitute the dark matter with lepton numbers $L=0,0.003,0.01$. 0.1. See Abazajian (2009) for explanation of the various observational constraints that come from Tremaine \& Gunn (1979); Bode et al. (2001); Boyarsky et al. (2006); Strigari et al. (2006); Abazajian et al. (2007). Right: most recent X-ray constraints (reproduced trom Horiuchi et al. (2014)), based on deep (Chandra (Horluchi et al. 2014) and XMM-Newton (Watson et al. 2012) observations of M31 and Suzaku observations of Ursa Minor (Loewenstein et al. 2009). The red band marked "Dodenson \& Widrow" is same as the $L=0$ curve in left panel. Our measurement lays at the boundary of the constraints from M31.

our Section 1). The mass of the sterile neutrino would be double the decay photon energy, $m_{s}=7.1 \mathrm{keV}$. The line flux detected in our full sample corresponds to a mixing angle for the decay $\sin ^{2}(2 \theta) \sim 7 \times 10^{-11}$. This value is below the upper limits placed by the previous searches, shown in Figure 13. Our detections from the stacked XMM-Newton MOS observations of galaxy clusters are shown with a star in red in that figure. Figure 14 shows the detections and upper limits we obtained from our various subsamples we used in this work (based on the included cluster masses and distances), as well as a comparison with the previous upper limit placed using the Bullet cluster by Boyarsky et al. (2008) at $3.57 \mathrm{keV}$, which is the most relevant earlier constraint for us. Since the mixing angle is a universal quantity, all the subsample measurements must agree.

The line in the subsample of 69 fainter clusters (full sample sans Perseus, Coma, Ophiuchus and Centaurus) corresponds to a mixing angle that is consistent with the full sample; the same is seen (though with a mild $1.5 \sigma$ tension) for the subsample of bright nearby clusters Coma+Centaurus+Ophiuchus. However, the brightness of the new line in the XMM-Newton spectrum of Perseus corresponds to a significantly higher mixing angle than that for the full sample (by factor of eight in terms of the line flux for the MOS spectrum), which poses a problem in need of further investigation. In principle, an enhanced flux of the detected line in the Perseus spectra may be due to a dark matter filament along the line of sight, though it would have to be rather extreme, so it is wise to look for more plausible explanations.

We tried to excise the central $1^{\prime}$ region of the Perseus cluster, to see if the flux originates in the cool core of the cluster. Indeed, this decreased the flux in the line in half and removed most of the tension with the other measurements. However, this suggests that either some of the line flux is astrophysical in origin (at least in Perseus), or the cool gas in the core of the cluster affects our ability to measure the continuum and the fluxes of the nearby $\mathrm{K}$ XVIII and Ar XVII lines, in the end resulting in an overestimate of the flux of our detected line. It appears that in Perseus, there is an anomalously strong line at the position of the Ar XVII DR line at $3.62 \mathrm{keV}$.

With this knowledge, we have tried to add this anomalous $3.62 \mathrm{keV}$ line in the model for the full sample, where we have the most statistically significant line detection. The additional line is still required, albeit at a lower significance and a slightly lower energy of $3.55 \pm 0.03 \mathrm{keV}$. Note that the sample of bright clusters is dominated by the emission from the cool cores of the Ophiuchus and Centaurus Clusters, if this Ar $3.62 \mathrm{keV}$ line anomaly is typical of cool cores, they may also be affected. However, freeing the flux of the $3.62 \mathrm{keV}$ line in the MOS fullsample fit did not require additional contribution from clusters other than Perseus, though the constraints are obviously weak.

The radial distribution of the flux of this line should be investigated further in the nearby bright clusters, including those with and without cool cores.

We note that even if the sterile neutrino interpretation of the emission line is correct, this detection would not necessarily imply that all dark matter is composed of these particles. Assuming a standard cosmological history below a temperature of a few hundred $\mathrm{MeV}$, sterile 


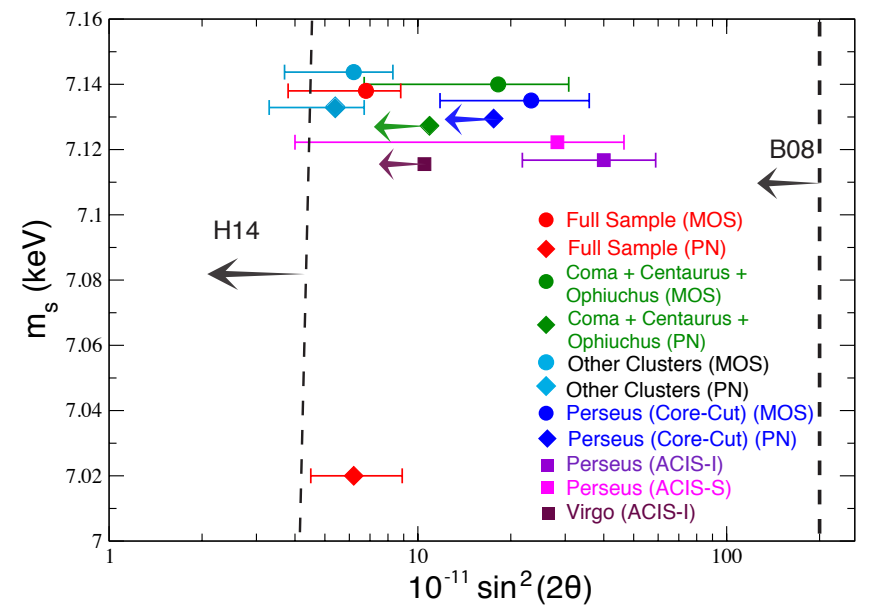

Figure 14. Sterile neutrino mass and mixing angle measurements and upper limits obtained from the different samples used in this study. The comparison of our stacking method with the limits placed by the single well-exposed Bullet Cluster at $3.57 \mathrm{keV}$ Boyarsky et al. (2008) and Horiuchi et al. (2014) are also shown and marked with "B08" and "H14" in the higure, respectively. The error bars and upper limits are in the $90 \%$ confidence level.

neutrinos would be produced by oscillations with active neutrinos at an abundance determined by the mass and mixing angle (e.g. Dodelson \& Widrow 1994 Kusenko 2009). Accounting for the increase in mixing angle that would be inferred for a dark matter fraction in sterile neutrinos less than unity, we find that this fraction is $\sim 13 \%-19 \%$ based on the methods in Abazajian (2006) and Asaka et al. (2007) - and cannot exceed 26\% based on the absolute lower bound distorted wave production estimate in Asaka et al. (2007).

This implies that either (1) sterile neutrinos are a subdominant component of dark matter, (2) sterile neutrinos are predominantly produced by some other mechanism, or (3) the emission line originates from some other radiatively decaying light dark matter candidate such as moduli dark matter (Kusenko et al. 2013). The Shi-Fuller mechanism is one of the possible production mechanisms for the sterile neutrino dark matter interpretation of this detection. The implications of the detection for structure formation in cosmological small scales are discussed in detail in (Abazajian 2014).

They may also be produced by means that do not involve oscillations, such as inflaton or Higgs decay (Kusenko 2006: Shaposhnikov \& Tkachev 2006: Petraki \& Kusenko 2008; Kusenko 2009), although there may still be sufficient mixing to provide an observable radiative decay signal. This detection is consistent with $100 \%$ of dark matter composed of sterile neutrinos produced by these mechanisms, as well as by the split seesaw mechanism (Kusenko, Takahashi, \& Yanagida 2010). Even in this case, some sterile neutrinos would be produced by non-resonant oscillations. However, based again on the calculations in Abazajian (2006) and Asaka et al. (2007), only $\sim 1 \%-3 \%$ of the sterile neutrino abundance (with an upper limit of $7 \%$ ) would be accounted for in this way for a sterile neutrino with mass of $7.1 \mathrm{keV}$ and a mixing angle corresponding to $\sin ^{2}(2 \theta) \sim 7 \times 10^{-11}$.

Our result must be verified using a variety of X-ray instruments, X-ray emitting dark matter dominated objects, methods of data reduction, background subtrac-

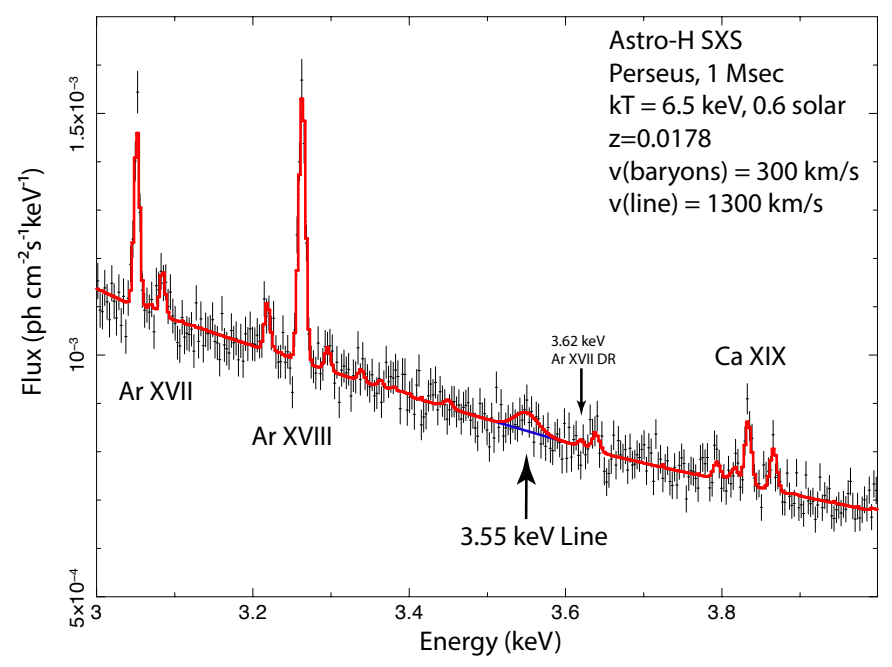

Figure 15. $1 \mathrm{Ms}$ Astro-H Soft X-ray Spectrometer (SXS) simulations of the Perseus Cluster. The line width corresponds to line of sight velocity dispersion of $1300 \mathrm{~km} \mathrm{~s}^{-1}$. The figure shows that the decaying dark matter line broadened by the virial velocities of dark matter particles will easily be distinguished from the plasma emission lines which are broadened by turbulence in sufficiently deep observations of the Perseus Cluster.

tion, and statistical techniques to investigate the interpretation of this line. The future high-resolution Astro- $\mathrm{H}$ observations will be able to measure the broadening of the line, which will allow us to measure its velocity dispersion. To detect a dark matter decay line, which is much weaker than the plasma lines will require a significantly long exposure. We performed $1 \mathrm{Ms}$ Astro-H SXS simulations of the Perseus Cluster assuming that the width $(15 \mathrm{eV})$ of the dark matter decay line is determined by the virial velocities of dark matter particles of $1300 \mathrm{~km} \mathrm{~s}^{-1}$. Figure 15 shows that the broader dark matter line will be easily distinguished from the plasma emission lines, which are only broadened by the turbulence in the X-ray emitting gas.

\section{CAVEATS}

As intriguing as the dark matter interpretation of our new line is, we should emphasize the significant systematic uncertainties affecting the line energy and flux in addition to the quoted statistical errors. The line is very weak, with an equivalent width in the full-sample spectra of only $\sim 1 \mathrm{eV}$. Given the CCD energy resolution of $\sim 100 \mathrm{eV}$, this means that our line is a $\sim 1 \%$ bump above the continuum. This is why an accurate continuum model in the immediate vicinity of the line is extremely important; we could not leave even moderately significant residuals unmodeled. To achieve this, we could not rely on any standard plasma emission models and instead had to let all the tabulated lines free (including their fluxes, energies and widths, within reasonable bounds), as described in Section 3.

This approach results in a very large number of parameters to fit simultaneously, among which are the line energies and widths that notoriously cause problems for the statistic minimization algorithms. It was difficult to make XSPEC find absolute minima; the convergence of all of the reported fits had to be verified by manually varying key parameters and refitting using different minimization algorithms. Nevertheless, it is not incon- 
ceivable that some of our fits did not find an absolute $\chi^{2}$ minimum.

Another consequence of the very low line flux is illustrated by the lower panels in Figure 5, showing the effective area curves. There are variations in these curves at a $\sim 1 \%$ level, some of which may be at the position of our line. If these features are not accurately calibrated, they may cause artifacts that would be falsely detected as spectral features. This is where our stacking approach makes a big difference - those effective area variations are almost completely smeared out in the "all other" sample, for which the instrument response was averaged over a large range of cluster redshifts (lowest panels), and the line is still present in those spectra. Nevertheless, for the nearby subsamples, these variations represent a systematic uncertainty.

Finally, there is a discrepancy, albeit at a marginal statistical significance, between the line energy obtained for the full sample by MOS and PN (see Table 5). The energies come into agreement if we allow the flux of the Ar $3.62 \mathrm{keV}$ line in the MOS full-sample spectrum to increase many times above its theoretical upper limit, to a value corresponding to that observed in Perseus (which is part of the full sample); see Figure 9. (The Perseus contribution to the PN full sample is smaller, and the $3.62 \mathrm{keV}$ line would not affect the fit.) The new line in MOS becomes slightly fainter and less statistically significant, with a lower energy. However, the energy of both MOS and PN lines then becomes consistent with that of the weak K line at $3.515 \mathrm{keV}$. The expected flux of the $\mathrm{K}$ line (Table 5) is 10-20 times lower than the observed line fluxes for both detectors; if something is wrong by that much in the thermal plasma model, this would provide an alternative explanation for our line. The MOS full-spectrum line is also consistent with just the 3.62 $\mathrm{keV}$ line at the anomalously high flux that accounts for Perseus, if the new line is at the higher end of its error interval. However, this would not explain the PN line.

Disentangling these possibilities is impossible at the present energy resolution and has to wait until the launch of Astro- $H$. The other systematic uncertainties mentioned above also have the low-energy resolution as their root cause.

In the meantime, we are planning to analyze other independent stacked samples from Chandra and Suzaku and their various subsamples. In particular, it would be very interesting to pinpoint the origin, if not the nature, of the anomalous Ar $3.62 \mathrm{keV}$ line. We will also divide the clusters spatially into annuli, to study the radial distribution of the emission and determine if the flux is proportional to the mass density squared, in order to distinguish the thermal plasma emission from a dark matter decay line.

Soon after this manuscript has been submitted and posted on the preprint archive, a preprint by Boyarsky et al. (2014) was posted, which reported an independent detection of a line at the same energy in the XMM-Newton spectra of M31 and the outskirts of the Perseus Cluster. They excluded the central Perseus pointing that we used in our XMM-Newton analysis; thus, their dataset has no overlap with ours. Their X-ray line flux, when converted to the sterile neutrino decay rate, is in agreement with ours, providing an independent confirmation of the line existence (and perhaps even of its dark matter origin).

\section{ACKNOWLEDGEMENTS}

The authors thank Alexey Vikhlinin for extensive discussions, cross-checking the results and spotting several errors; Douglas Finkbeiner and Serkan Cabi for useful discussions; Christine Jones, Signe Riemer-Sorensen, Alexander Kusenko, and the anonymous referee for useful comments on the draft; and Kevork Abazajian, and Shunsaku Horiuchi for providing the limits from their M31 analysis. E.B. also thanks Adrian Batu Gerard for patiently waiting for the submission to be born. EB was supported in part by Chandra grant AR0-11020X and GO1-12104X. A.F. and R.S. were supported in part by NASA ADAP grant NNX12AF44G. S.W.R. was supported by the Chandra X-ray Center through NASA contract NAS8-03060, and the Smithsonian Institution.

\section{REFERENCES}

Abazajian, K., Fuller, G. M., \& Patel, M. 2001, Phys. Rev., 64, 023501

Abazajian, K., Fuller, G. M., \& Tucker, W. H. 2001, ApJ, 562, 593

Abazajian, K. 2006, Phys. Rev., 73, 063506

Abazajian, K. N., Markevitch, M., Koushiappas, S. M., \& Hickox, R. C. 2007, Phys. Rev., 75, 063511

Abazajian, K. 2009, astro2010: The Astronomy and Astrophysics Decadal Survey, 2010, 1

Abazajian, K. N., Acero, M. A., Agarwalla, S. K., et al. 2012, arXiv:1204.5379

Abazajian, K. N. 2014, arXiv:1403.0954

Anders, E., \& Grevesse, N. 1989, Geochimica et Cosmochimica Acta, 53, 197

Arnaud, K. A. 1996, Astronomical Data Analysis Software and Systems V, 101, 17

Asaka, T., Shaposhnikov, M., \& Laine, M. 2007, Journal of High Energy Physics, 1, 91

Bartelmann, M. 1996, A\&A, 313, 697

Bode, P., Ostriker, J. P., \& Turok, N. 2001, ApJ, 556, 93

Böhringer, H., Voges, W., Huchra, J. P., et al. 2000, ApJS, 129, 435

Böhringer, H., \& Werner, N. 2010, A\&A Rev., 18, 127

Böhringer, H., Schuecker, P., Guzzo, L., et al. 2004, A\&A, 425, 367

Boyarsky, A., Neronov, A., Ruchayskiy, O., Shaposhnikov, M., \&

Tkachev, I. 2006, Physical Review Letters, 97, 261302

Boyarsky, A., Neronov, A., Ruchayskiy, O., \& Shaposhnikov, M. 2006, MNRAS, 370, 213

Boyarsky, A., Ruchayskiy, O., \& Markevitch, M. 2008, ApJ, 673, 752

Boyarsky, A., Ruchayskiy, O., \& Shaposhnikov, M. 2009, Annual Review of Nuclear and Particle Science, 59, 191

Boyarsky, A., Ruchayskiy, O., Iakubovskyi, D., et al. 2010, MNRAS, 407, 1188

Boyarsky, A., Iakubovskyi, D., \& Ruchayskiy, O. 2012, Physics of the Dark Universe, 1, 136

Boyarsky, A., Ruchayskiy, O., Iakubovskyi, D., \& Franse, J. 2014, arXiv:1402.4119

Bulbul, G. E., Hasler, N., Bonamente, M., \& Joy, M. 2010, ApJ, 720,1038

Bulbul, G. E., Smith, R. K., Foster, A., et al. 2012a, ApJ, 747, 32

Bulbul, E., Smith, R. K., \& Loewenstein, M. 2012b, ApJ, 753, 54

Clowe, D., Bradač, M., Gonzalez, A. H., et al. 2006, ApJL, 648, L109

Dodelson, S., \& Widrow, L. M. 1994, Physical Review Letters, 72, 17

Ebeling, H., Voges, W., Bohringer, H., et al. 1996, MNRAS, 281, 799

Ebeling, H., Edge, A. C., Allen, S. W., et al. 2000, MNRAS, 318, 333

Ebeling, H., Edge, A. C., \& Henry, J. P. 2001, ApJ, 553, 668

Foster, A. R., Ji, L., Smith, R. K., \& Brickhouse, N. S. 2012, ApJ, 756,128

Golse, G., \& Kneib, J.-P. 2002, A\&A, 390, 821

Gonzalez, A. H., Sivanandam, S., Zabludoff, A. I., \& Zaritsky, D. 2013, arXiv:1309.3565

Hickox, R. C., \& Markevitch, M. 2006, ApJ, 645, 95

Hinshaw, G., Larson, D., Komatsu, E., et al. 2013, ApJS, 208, 19 
Horiuchi, S., Humphrey, P. J., Oñorbe, J., et al. 2014, Phys . Rev., 89, 025017

Kuntz, K. D., \& Snowden, S. L. 2008, A\&A, 478, 575

Kusenko, A. 2006, Physical Review Letters, 97, 241301

Kusenko, A. 2009, PhysRep, 481, 1

Kusenko, A., Takahashi, F., \& Yanagida, T. T. 2010, Phys. Lett. B, 693, 144

Kusenko, A., Loewenstein, M., \& Yanagida, T. T. 2013, Phys. Rev., 87, 043508

Lin, Y.-T., \& Mohr, J. J. 2004, ApJ, 617, 879

Loewenstein, M., Kusenko, A., \& Biermann, P. L. 2009, ApJ, 700, 426

Loewenstein, M. 2013, ApJ, 773, 52

Mitchell, R. J., Culhane, J. L., Davison, P. J. N., \& Ives, J. C. 1976, MNRAS, 175, 29P

Navarro, J. F., Frenk, C. S., \& White, S. D. M. 1997, ApJ, 490, 493

Paerels, F. B. S., \& Kahn, S. M. 2003, ARAA, 41, 291

Pal, P. B., \& Wolfenstein, L. 1982, Phys. Rev., 25, 766

Palmeri, P., Quinet, P., Mendoza, C., et al. 2012, A\&A, 543, A44

Petraki, K., \& Kusenko, A. 2008, Phys. Rev., 77, 065014

Protassov, R., van Dyk, D. A., Connors, A., Kashyap, V. L., \& Siemiginowska, A. 2002, ApJ, 571, 545

Riemer-Sørensen, S., Hansen, S. H., \& Pedersen, K. 2006, ApJL, 644, L33

Riemer-Sørensen, S., \& Hansen, S. H. 2009, A\&A, 500, L37

Sanders, J. S., \& Fabian, A. C. 2011, MNRAS, 412, L35

Shaposhnikov, M., \& Tkachev, I. 2006, Physics Letters B, 639, 414 A. 2008, Phys. Rev., 77, 065014
Serlemitsos, P. J., Smith, B. W., Boldt, E. A., Holt, S. S., \& Swank, J. H. 1977, ApJL, 211, L63

Shi, X., \& Fuller, G. M. 1999, Physical Review Letters, 83, 3120

Smith, R. K., Brickhouse, N. S., Liedahl, D. A., \& Raymond, J. C. 2001, ApJL, 556, L91

Smith, R. K., Foster, A. R. \& Brickhouse, N. S. 2012, Astronomische Nachrichten, 333, 301

Snowden, S. L., Mushotzky, R. F., Kuntz, K. D., \& Davis, D. S. 2008, A\&A, 478, 615

Strigari, L. E., Bullock, J. S., Kaplinghat, M., et al. 2006, ApJ, 652,306

Tamura, T., Maeda, Y., Mitsuda, K., et al. 2009, ApJL, 705, L62

Tremaine, S., \& Gunn, J. E. 1979, Physical Review Letters, 42, 407

Watson, C. R., Li, Z., \& Polley, N. K. 2012, JCAP, 3, 18

Werner, N., de Plaa, J., Kaastra, J. S., et al. 2006, A\&A, 449, 475

Vainshtein, L. A., \& Safronova, U. I. 1980, ADNDT, 25, 311

Vikhlinin, A., Markevitch, M., Murray, S. S., et al. 2005, ApJ, 628,655

Vikhlinin, A., Kravtsov, A., Forman, W., et al. 2006, ApJ, 640, 691

Vikhlinin, A., Burenin, R. A., Ebeling, H., et al. 2009, ApJ, 692, 1033

Zwicky, F. 1933, Helvetica Physica Acta, 6, 110

Zwicky, F. 1937, ApJ, 86, 217 